\title{
Increased Spontaneous Firing Rates in Auditory Midbrain Following Noise Exposure Are Specifically Abolished by a Kv3 Channel Modulator
}

Lucy A. Anderson ${ }^{1, \star}$, Lara L. Hesse ${ }^{1,2, \star}$, Nadia Pilati ${ }^{3, \star}$, Warren M.H. Bakay ${ }^{1}$, Giuseppe Alvaro $^{3}$, Charles H. Large ${ }^{4}$, David McAlpine ${ }^{1, \$}$, Roland Schaette ${ }^{1}$, and Jennifer F. Linden ${ }^{1,5, \$}$ *these authors contributed equally to the work

${ }^{1}$ Ear Institute, University College London, 332 Gray's Inn Road, London, WC1X 8EE, UK ${ }^{2}$ Department for Otolaryngology, Head and Neck Surgery, University of Lübeck, Ratzeburger Allee 160, 23538 Lübeck, Germany

${ }^{3}$ Autifony SRL, Via Ugo Bassi 58b, University of Padova, 35121 Padova, Italy

${ }^{4}$ Autifony Therapeutics Ltd., Stevenage Biosciences Catalyst, Gunnels Wood Road, Stevenage, SG1 2FX, UK

${ }^{5}$ Department of Neuroscience, Physiology \& Pharmacology, University College London, Gower Street, London, WC1E 6BT, UK

§Present address: Australian Hearing Hub, Macquarie University, 16 University Avenue, NSW 2109, Australia

${ }^{\$}$ To whom correspondence should be addressed: Dr Jennifer F. Linden, UCL Ear Institute, 332 Gray's Inn Road, London, WC1X 8EE, UK. Email: j.linden@ucl.ac.uk 


\section{Abstract}

Noise exposure has been shown to produce long-lasting increases in spontaneous activity in central auditory structures in animal models, and similar pathologies are thought to contribute to clinical phenomena such as hyperacusis or tinnitus in humans. Here we demonstrate that multi-unit spontaneous neuronal activity in the inferior colliculus (IC) of mice is significantly elevated four weeks following noise exposure at recording sites with frequency tuning within or near the noise exposure band, and this selective central auditory pathology can be normalised through administration of a novel compound that modulates activity of Kv3 voltage-gated ion channels. The compound had no statistically significant effect on IC spontaneous activity without noise exposure, nor on thresholds or frequency tuning of toneevoked responses either with or without noise exposure. Administration of the compound produced some reduction in the magnitude of evoked responses to a broadband noise, but unlike effects on spontaneous rates, these effects on evoked responses were not specific to recording sites with frequency tuning within the noise exposure band. Thus, the results suggest that modulators of $\mathrm{Kv} 3$ channels can selectively counteract increases in spontaneous activity in the auditory midbrain associated with noise exposure.

\section{Keywords}

inferior colliculus, noise exposure, acoustic trauma, potassium channels, spontaneous activity, AUT00063

\section{Highlights}

- Spontaneous activity in mouse inferior colliculus is elevated after noise exposure

- AUT00063, a novel Kv3 channel modulator, normalises this midbrain pathology

- No effect of AUT00063 on IC spontaneous activity without noise exposure

- No effect of AUT00063 on IC tone-evoked response thresholds or frequency tuning 


\section{Introduction}

Animal studies have identified increased spontaneous firing rates along the auditory pathway as a putative neurophysiological correlate of central auditory pathologies associated with noise exposure in humans, such as hyperacusis and tinnitus (Roberts et al., 2010; Norena, 2011; Auerbach et al., 2014; Brotherton et al., 2015; Eggermont and Roberts, 2015; Norena, 2015). Studies of rodent cochlear nucleus (Kaltenbach et al., 1998; Brozoski et al., 2002; Kaltenbach et al., 2004; Finlayson and Kaltenbach, 2009; Vogler et al., 2011; Dehmel et al., 2012; Koehler and Shore, 2013; Wu et al., 2016; Glait et al., 2018), inferior colliculus (Mulders and Robertson, 2009; Mulders et al., 2011; Manzoor et al., 2012; Manzoor et al., 2013; Mulders et al., 2014; Vogler et al., 2014), auditory thalamus (Kalappa et al., 2014) and auditory cortex (Engineer et al., 2011; Ahlf et al., 2012) have reported significant elevations in spontaneous firing rates in these structures for many weeks following noise exposure. This spontaneous hyperactivity in central auditory structures could arise as a consequence of decreased auditory nerve activity following cochlear damage; homeostatic plasticity mechanisms may act to stabilise the mean firing rate by increasing neuronal response gain, producing elevated spontaneous firing rates and, potentially, auditory phantom perceptions (Schaette and Kempter, 2006; Norena, 2011). Computational modelling points to a putative relationship between central auditory hyperactivity and increased neuronal gain (Schaette and Kempter, 2006, 2008; Chrostowski et al., 2011; Norena, 2011). Moreover, neuronal hyperactivity in the inferior colliculus (IC) depends on input from the cochlea and dorsal cochlear nucleus (Mulders and Robertson, 2009; Manzoor et al., 2012; Robertson et al., 2013), suggesting that hyperactivity might be generated through amplification of spontaneous activity along the auditory pathway by means of increased neuronal response gain. While the mechanisms of such central amplification remain unclear and may be complex, it seems possible that elevated spontaneous firing rates in the auditory midbrain following noise exposure might be reduced through manipulations that decrease central 
auditory hyperactivity, for example by increasing inhibition, reducing excitation, or decreasing intrinsic neural excitability.

Voltage-gated potassium channels of the Kv3 family are potential targets for attempts to decrease central auditory hyperactivity and counteract the pathological effects of noise exposure. Kv3.1 and Kv3.3 channels are highly expressed in auditory brainstem nuclei responsive to high temporal frequencies (Gan and Kaczmarek, 1998; Grigg et al., 2000; Li et al., 2001). Kv3 currents are high voltage activated (HVA), requiring depolarisation achieved only during an action potential. By activating at depolarised potentials and rapidly deactivating, Kv3 currents facilitate action potential repolarisation, promoting regular and accurate sound-evoked firing (Rudy et al., 1999).

Expression and function of Kv3.1 channels in the auditory system are dynamically affected by sound exposure (Strumbos et al., 2010) and reduced expression of these channels has been associated with age-related hearing loss (von Hehn et al., 2004). Down-regulation of Kv-like HVA currents was previously reported in the dorsal cochlear nucleus (DCN) shortly after noise exposure, and appeared to be correlated with the emergence of abnormal burst firing in DCN principal neurons, the fusiform cells (Pilati et al., 2012). Thus, down-regulation of Kv3 channel expression following noise exposure is hypothesised to alter neuronal processing in the central auditory system, potentially contributing to an increase in central auditory responsiveness which could generate hearing pathologies associated with noise exposure. The effects of Kv3 channel modulators on central auditory neurons have recently been studied in vitro (Brown et al., 2016) and in vivo in a mouse model of auditory neuropathy (Chambers et al., 2017). The present study in inferior colliculus, and related recently published work by Glait et al. (2018) in cochlear nucleus, are the first published reports on the in vivo effects of Kv3 channel modulators on central auditory processing following noise exposure. 
Here we investigate the effects of administration of AUT00063 (Autifony Therapeutics Limited), a modulator of Kv3 potassium channels, on auditory midbrain activity in vivo following noise exposure in mice. AUT00063 produces a leftward shift in the voltagedependence of activation of Kv3.1 and Kv3.3 channels, presumably by increasing the open probability of the channels (cf. Brown et al., 2016). Consequently, AUT00063 enhances Kv3mediated currents evoked by depolarisation from neuronal resting potentials, potentially counteracting noise-induced down-regulation of these currents in the auditory brainstem. An AUT00063-induced shift in the activation of Kv3 channels toward more negative potentials could lead to earlier activation of Kv3 channels during the rising phase of an action potential and later deactivation during action potential repolarisation, increasing action potential afterhyperpolarisation amplitude and potentially preventing the initiation of burst firing (cf. Chambers et al., 2017). We therefore postulated that AUT00063 might normalize neuronal hyperactivity in the auditory brainstem following noise exposure.

To test this hypothesis, we exposed mice to octave-band (8-16 kHz) noise at $105 \mathrm{~dB}$ SPL for 2 hours, and then recorded neural activity in the inferior colliculus four weeks later. We observed an increase in spontaneous firing rates at recording sites with characteristic frequencies within and slightly above the noise exposure frequency range. Administration of AUT00063 reduced this spontaneous hyperactivity back to control levels without affecting tone-evoked response thresholds or frequency tuning. AUT00063 administration also produced some reduction in the magnitude of responses evoked by a broadband noise stimulus, but unlike the reduction in spontaneous firing rates, this effect was not specific to multi-unit recordings tuned near the noise exposure frequency range. The results suggest that Kv3 modulators might specifically counteract pathological increases in spontaneous firing rates in the auditory midbrain following noise exposure. 


\section{Methods}

\section{Pharmacology}

Interaction of AUT00063 with recombinant Kv3 potassium channels expressed in mammalian cell lines was investigated using patch-clamp electrophysiology techniques. Interaction of AUT00063 with other pharmacological targets was investigated in a series of functional and ligand binding "cross screening" assays (Scinovo-GSK, UK; CEREP, France).

\section{Recombinant Channel Electrophysiology}

\section{Cell culture}

IonWorks assay. CGE22 frozen cells pre-transduced with hKv3.1a (NM_001112741.1) BacMam were used. Cells (5 to 10 million) were plated in DMEM/F12 complete Dulbecco's modified Eagle's medium F-12 medium (Gibco) supplemented with 10\% heat inactivated fetal bovine serum (PAA), 1\% penicillinstreptomycin (Invitrogen), 1\% L-gluatmine (Invitrogen), 1\% non-essential aminoacids (PAA). Culturing conditions: $37^{\circ} \mathrm{C}$ under $5 \% \mathrm{CO} 2$ for 24 hours and $30^{\circ} \mathrm{C}$ under $5 \% \mathrm{CO} 2$ for $18-24$ hours.

Q-Patch assay. BacMam-transduced U2OS cells expressing hKv3.1a channels were used. They were transduced by incubation overnight ( 8 hours) with $1 \%$ of a BacMam-hKv3.1a or hKv3.2a reagent $(2.3 \times 108 \mathrm{pfu} / \mathrm{ml})$. Cells were re-suspended in fresh 293 SFM II medium (Gibco) containing $25 \mathrm{mM}$ HEPES (Gibco) and $0.04 \mathrm{mg} / \mathrm{ml}$ soybean trypsin inhibitor (SigmaAldrich) and transferred to a QPatch recording station.

\section{Voltage-clamp recordings}

IonWorks assay. The population patch-clamp mode of automated voltage clamp recording with IonWorks QuattroTM (Molecular Devices, LLC, Sunnyvale, CA, USA) was used. Briefly, effects of AUT00063 on hKv3.1a channels were tested using 384-well population patchclamp plates. Seal resistance was measured for each well and cells were perforated by 
incubation with $100 \mu \mathrm{g} / \mathrm{mL}$ amphotericin B (Sigma-Aldrich). Cells were held at -70 mV, stepped to $-15 \mathrm{mV}$ for $100 \mathrm{~ms}$ (partial channel activation) and after $100 \mathrm{~ms}$ at $-70 \mathrm{mV}$ a second pulse to $+40 \mathrm{mV}$ was applied for $50 \mathrm{~ms}$ (full channel activation). In all experiments, this voltage protocol was applied to cells before and following a 3 minute incubation with AUT00063. AUT00063 was tested at different concentrations ranging from 50nM up to $50 \mu \mathrm{M}$ (11 points per curve). 1-cyclohexyl-1-[(7,8-dimethyl-2-oxo-1H-quinolin-3-yl)methyl]-3phenylurea $(10 \mu \mathrm{M}$, Aurora Screening Library), which we had previously found to be a potent and full activator of human Kv3.1 and Kv3.2 channels, was included in all assays as a standard. External buffer with the addition of dimethyl sulfoxide (0.5\% DMSO, Sigma-Aldrich) was also tested to provide a vehicle baseline. Recordings were performed in the following buffers: Dulbecco's PBS with $0.5 \mathrm{mM} \mathrm{MgCl}_{2}$ and $0.9 \mathrm{mM} \mathrm{CaCl}_{2}$ (Invitrogen) as extracellular solution and $50 \mathrm{mM} \mathrm{KCl}, 100 \mathrm{mM} \mathrm{K}$-gluconate, $3.2 \mathrm{mM} \mathrm{MgCl}_{2}$, and $5 \mathrm{mM} \mathrm{HEPES,} \mathrm{pH} 7.3$ adjusted with $\mathrm{KOH}$ as intracellular solution (Sigma-Aldrich). An online correction of $+15-20$ $\mathrm{mV}$ was applied to correct for junction potentials. The current signal was sampled at $10 \mathrm{kHz}$.

Q-Patch assay. Patch-clamp recordings where greater voltage control was required were performed using the automated recording system QPatch-16TM (Sophion Bioscience, Denmark). Cells were suspended in extracellular solution containing: $145 \mathrm{mM} \mathrm{NaCl}, 4 \mathrm{mM}$ $\mathrm{KCl}, 1 \mathrm{mM} \mathrm{MgCl} 2,2 \mathrm{mM} \mathrm{CaCl} 2,10 \mathrm{mM}$ glucose and 10mM HEPES (solution by GE Healthcare); $\mathrm{pH} 7.3$ adjusted with $\mathrm{NaOH}$, and whole-cell recordings were carried out using an intracellular solution (Sigma-Aldrich) containing: 10mM NaCl, 100mM KGluconate, 10mM HEPES, 10mM EGTA and 32mM KF; pH 7.2 adjusted with KOH. Cell membrane potentials were held at $-90 \mathrm{mV}$ and currents were evoked by voltage steps (200 ms duration) from -60 $\mathrm{mV}$ to $+30 \mathrm{mV}$ (in $10 \mathrm{mV}$ increments). Vehicle (0.1\% DMSO) or 10 $\mu \mathrm{M}$ AUT00063 were applied for at least 3 minutes prior to the subsequent voltage step protocol application. A P/4 leak subtraction protocol was applied, and serial resistance values were constantly monitored. Any cell where serial resistance exceeded $20 \mathrm{M} \Omega$ was eliminated from the subsequent analysis. 


\section{Data analysis}

IonWorks assay. Paired comparisons between pre- and post-drug additions of steady-state currents measured at $-15 \mathrm{mV}$ voltage step were used to determine the effect of the compound. Concentration-response data were normalised to the maximum effect (i.e. 100\%) observed with the standard. Normalised data were then plotted against the concentration of the compound and analysed using GraphPad Prism software 6.03 (GraphPad Software, Inc., CA, USA). The log of concentrations required to increase current by 50\% (pEC50) was determined by fitting data points to the four-parameter logistic function (sigmoidal doseresponse) where Bottom is the $Y$ value at the bottom plateau, Top is the $Y$ value at the top plateau, and pEC50 is the $X$ value (log concentration) when the response is halfway between Bottom and Top. Tau deactivation values were calculated by fitting the decaying phase of the tail currents recorded at $-40 \mathrm{mV}$ following a $50 \mathrm{~ms}$ pulse at $+40 \mathrm{mV}$ to an exponential function. Only cells with a mean current amplitude at $+40 \mathrm{mV}$ greater than $0.5 \mathrm{nA}$ and a seal resistance less than $20 \mathrm{M} \Omega$ were included in the data analysis.

Q-Patch assay. Data analysis was performed using Sophion's QPatch assay software in combination with GraphPad Prism software 6.03 and Microsoft Excel ${ }^{\mathrm{TM}}$ (Redmond, WA, USA). Potentiation of the currents was calculated as $\%$ potentiation $=$ [current $(c p d) /$ current $(v e h)]^{\star} 100$; where current $(c p d)$ was the amplitude of the steady-state current in the presence of the compound and current (veh) was the amplitude in the presence of vehicle. For voltage dependence of activation experiments, normalised conductance-voltage plots were obtained by normalising conductance $(\mathrm{G})$ to maximal conductance (Gmax) and fit using the Boltzmann isoform $\mathrm{G}=\mathrm{Gmax} /\left[1+\exp \left(\left(\mathrm{V}^{-} \mathrm{V}^{1 / 2}\right) / \mathrm{k}\right)\right]$, where $\mathrm{V}^{1 / 2}$ is the voltage at half-maximal activation and $\mathrm{k}$ is the slope factor. Conductance values were obtained by dividing the current by the $\mathrm{K}+$-electrochemical driving force $(\mathrm{IK} /(\mathrm{Vm}-\mathrm{EK}))$. An unpaired $t$-test was performed to compare the activation $\mathrm{V}^{1} \frac{1}{2}$ and $\mathrm{k}$ during the control period and after AUT00063 
application. Results are reported as mean \pm s.e.m. Differences are reported as significant where $p<0.05$.

\section{Pharmacokinetics}

The pharmacokinetics study was designed to assess the pharmacokinetics of AUT00063 in the male CBA/Ca mouse following intraperitoneal dosing at $90 \mathrm{mg} / \mathrm{kg}$. Pharmacokinetics experiments were carried out by Aptuit (Verona, Italy) in accordance with national legislation regarding animal welfare (Italian Legislative Decree no. 26/2014 and European Directive no 2010/63/UE). All experiments were approved by the internal Aptuit Committee on Animal Research and Ethics under authorisation issued by the Italian Ministry of Health (Italian Ministry of Health Authorization Project No. 38200).

Four male CBA/Ca mice were obtained from Charles River Italy and used for experiments at age 15 weeks (weights 26, 26.1, 27 and $27.5 \mathrm{~g}$ ) to match mice used for in vivo electrophysiology experiments. Each received an intraperitoneal administration of AUT00063 at a target dose level of $90 \mathrm{mg} / \mathrm{kg}$. Blood samples were collected at intervals up to $2 \mathrm{~h}$ after dose administration. After i.p. administration, serial blood samples were collected via the caudal vein of each mouse, and then animals were sacrificed humanely. Blood sample collection was carried out in accordance with the recommendations of Diehl et al. (2001). Blood samples (50 $\mu \mathrm{L}$ at each time point for each animal) were collected at 10, 30, 60, 90 and 120 minutes after dosing, into potassium EDTA capillaries. A total of $250 \mu \mathrm{L}$ of blood was therefore collected from each animal within $2 \mathrm{~h}$. Animals were euthanised within $24 \mathrm{~h}$ of last blood sample. Following collection, each $50 \mu \mathrm{L}$ blood sample was placed on wet ice protected from light. Within $0.5 \mathrm{~h}$ of collection, a double $25 \mu \mathrm{L}$ aliquot was transferred into appropriately labelled micronic tubes and diluted with $75 \mu \mathrm{L}$ of Hepes $0.1 \mathrm{~N}$. Residual blood was discarded. Blood samples were frozen at approximately $-20^{\circ} \mathrm{C}$ as soon as possible after preparation and stored until analysis. Blood samples were assayed using a method based on protein precipitation with acetonitrile followed by HPLC/MS-MS analysis with an optimised 
analytical method. Since the stability of the analytes in blood is unknown, Calibration Standards (CS) and Quality Control samples (QC) were prepared on the day of dosing and stored together with study samples to balance for any possible analyte degradation. Study samples, CS, QC and blanks were spiked with an internal standard (IS) to improve the precision of the assay. Study samples were analysed together with CS, QC and blank samples (including also double blanks). From the calibration curve, the linear range of the analytical method was determined and lower and upper limits of quantitation specified. Blood concentration $(\mathrm{ng} / \mathrm{ml})$ was reported.

\section{In Vivo Electrophysiology}

\section{Subjects}

Twenty-one male CBA/Ca mice (12 noise-exposed and 9 control animals) were used to assess the effects of noise exposure and/or AUT00063 on neural activity in the inferior colliculus (IC). All mice were originally obtained from Charles River UK and were 7-19 weeks old at the time of noise exposure, with normal hearing recorded in auditory brainstem response measurements (see below). Control animals were age-matched littermates.

The original set of 21 animals included 6 noise-exposed animals used for experiments involving injection of AUT00063, 6 noise-exposed animals that received vehicle injection, 5 unexposed control animals that received AUT00063 injection, and 4 unexposed controls that received vehicle injection. Multi-unit IC recordings from 3 mice (2 noise-exposed, 1 control) failed tests for recording stability, and therefore all data from these mice were excluded from analysis (see Data analysis, below). The final in vivo electrophysiology data set included ABR and IC recordings from 10 noise-exposed animals (5 used for experiments involving injection of AUT00063, 5 vehicle) and 8 unexposed control animals (3 AUT00063, 5 vehicle).

All noise exposure and in vivo electrophysiology experiments were performed in the UK under a project licence approved by the UK Home Office (PPL 70/7202) in accordance with 
animal welfare regulations specified in the United Kingdom Animal (Scientific Procedures) Act of 1986.

\section{Noise exposure}

Mice were anaesthetised with ketamine and medetomidine (i.p., $10 \mathrm{ml} / \mathrm{kg}$ body weight of a cocktail of $10 \mathrm{mg} / \mathrm{ml}$ ketamine and $0.083 \mathrm{mg} / \mathrm{ml}$ medetomidine). Once anaesthetised, animals were placed in a custom-made sound-proof booth on a heated pad (Snugglesafe) maintained at approximately $38+/-2{ }^{\circ} \mathrm{C}$, directly underneath the centre of a speaker (Stage Line PA Horn Tweeter MHD-220N/RD) positioned $45 \mathrm{~cm}$ above the animal's head. Prior to each use, the speaker was calibrated (using a GRAS 40BF free-field microphone) to ensure that the frequency response was flat $( \pm 2 \mathrm{~dB})$ over the $8-16 \mathrm{kHz}$ range. Noise exposure was performed with an octave-band noise (8-16 kHz) at $105 \mathrm{~dB}$ SPL [RMS] for two hours. Noise stimuli were generated using an RX6 processor (Tucker-Davis Technologies, TDT), attenuated as required (TDT PA5) and amplified (TDT SA2). During the noise exposure, pedal reflex and breathing rate were checked every 30 min and additional anaesthesia given as required. After noise exposure, mice received one dose of atipamezole s.c. (2 ml/kg body weight, $0.5 \mathrm{mg} / \mathrm{ml}$ ) to aid recovery from anaesthesia. Control animals were age-matched mice that did not undergo this noise exposure procedure (nor a sham procedure).

\section{AUT00063 and vehicle preparation}

AUT00063 ((5R)-5-ethyl-5-methyl-3-[2-(\{4-methyl-3-[(trifluoromethyl)oxy]phenyl\}oxy)-5pyrimidinyl]-2,4imidazolidinedione, Autifony Therapeutics Limited) was administered as a suspension in 20\% w/w Captisol ${ }^{\circledR}$ (SBE-cyclodextrin) in 0.5\% w/v HPMC K15M containing $0.1 \% \mathrm{w} / \mathrm{v}$ Tween80M in sterile water. Vehicle solution lacked the AUT00063 compound but was otherwise identical. The drug or vehicle solution was made up fresh on the day of the experiment by a person other than the in vivo experimenter, so that the experimenter was blind as to whether drug or vehicle was administered on that day. 


\section{Auditory Brainstem Response (ABR) recordings}

For ABR recordings, animals were anaesthetised with an i.p. injection of ketamine and medetomidine (dosage as described above for noise exposure). Body temperature was maintained at $37.5 \pm 0.5^{\circ} \mathrm{C}$ using a homeothermic blanket (Harvard Apparatus). Subdermal electrodes (Rochester Medical) were inserted under the skin with the positive electrode positioned at the vertex, the negative electrode behind the ear ipsilateral to the speaker, and the ground electrode behind the ear contralateral to the speaker (which was blocked using a foam earplug). ABR signals were bandpass filtered $2.2 \mathrm{~Hz}-7.5 \mathrm{kHz}$ and amplified 20x (Tucker-Davis Technologies, TDT RA4LI) and digitised at $24,414 \mathrm{~Hz}$ sampling rate (TDT RA16SD), then additionally bandpass filtered in software for analysis (see Data analysis, below).

\section{Extracellular recordings in inferior colliculus (IC)}

Animals were anaesthetised with an i.p. injection of ketamine and medetomidine $(10 \mathrm{ml} / \mathrm{kg}$ body weight of a cocktail containing $10 \mathrm{mg} / \mathrm{ml}$ ketamine and $0.083 \mathrm{mg} / \mathrm{ml}$ medetomidine). Dexamethasone was also administered (10 $\mathrm{mg} / \mathrm{kg}$ body weight s.c.) to control edema and atropine sulphate $(0.05 \mathrm{mg} / \mathrm{kg}$ body weight s.c.) to reduce broncheal secretions. Lactated Ringers solution was given every two hours $(0.1 \mathrm{ml} \mathrm{s.c.})$ to maintain hydration. The animal's temperature was maintained at $37.5 \pm 0.5^{\circ} \mathrm{C}$ using a homeothermic blanket connected to a rectal thermistor. Once the pedal reflex had been abolished, the mouse was placed in a nose clamp to stabilise the head while leaving the ears free. To access the IC, a large craniotomy (4.5-6.5 mm posterior to Bregma, 0.5-3.5 mm lateral to midline) was performed on the righthand side, revealing the full surface of the right IC. Breathing rate was monitored throughout the surgery and at regular intervals during the subsequent neural recording procedure. Additional ketamine and medetomidine cocktail was administered as needed to maintain stable anaesthesia, either in bolus i.p. injections (typical dosage $30 \%$ of the initial dose) or by continuous infusion through an i.p. cannula. 
Extracellular recordings of multi-unit activity were made using single-shank silicon multielectrodes with 16 recording sites (NeuroNexus; 1x16 linear array, $100 \mu \mathrm{m}$ electrode spacing, $177 \mu \mathrm{m}^{2}$ electrode surface area, $\sim 1-2 \mathrm{M} \Omega$ impedance), which enabled sampling across the full extent of the depth of the central nucleus of the IC. Probes were positioned vertically with the tip touching the surface of the IC $5.2 \mathrm{~mm}$ posterior to Bregma and $1.5 \mathrm{~mm}$ right of midline, then advanced rapidly by $2000 \mu \mathrm{m}$ using a hydraulic microdrive (Neurocraft, FHC Inc.) and retracted rapidly by $\sim 400 \mu \mathrm{m}$ to minimise duration of tissue compression. Electrode signals were acquired at $24414 \mathrm{~Hz}$ sampling rate (TDT RA16SD and RX5), bandpass filtered typically $600-9000 \mathrm{~Hz}$, and thresholded with artifact rejection (Jan Schnupp's Brainware) to extract multi-unit activity. After initial characterisation of multi-unit spontaneous activity and auditory evoked responses at the recording site, the animal received a single i.p. injection of either AUT00063 at a dose of $90 \mathrm{mg} / \mathrm{kg}$ or an equivalent volume of vehicle. The characterisation of spontaneous and evoked multi-unit activity was repeated immediately after this injection and then at 30-minute intervals for up to 2 hours. Each pair of AUT00063 and vehicle experiments was carried out on age-matched animals on consecutive days, by an experimenter blind to the nature of the injection each day.

\section{$\underline{\text { Acoustic stimuli }}$}

For ABR recordings, stimuli were either $5 \mathrm{~ms}$ tone pips with a rise/fall time of $1.5 \mathrm{~ms}$ and sound frequencies of $8,11,16,22,32$, or $48 \mathrm{kHz}$, or 50 us clicks, both presented at sound intensities of 0-80 dB SPL in $5 \mathrm{~dB}$ steps. ABR stimuli (tone pips and clicks) were delivered in order of increasing sound intensity, with 500 repetitions of each parameter condition presented at a rate of 20 stimuli/second and an onset delay of $2 \mathrm{~ms}$.

For IC recordings, stimuli were tone pips and noise bursts. Tone parameters were: frequency range at least $4-70 \mathrm{kHz}$ in $1 / 8$ th octave steps, intensity range $0-80 \mathrm{~dB}$ SPL in $10 \mathrm{~dB}$ steps, duration $100 \mathrm{~ms}$ with $5 \mathrm{~ms}$ rise/fall cosine-squared gating. Tones were presented in randomised frequency-intensity order at an inter-tone interval of approximately $400 \mathrm{~ms}$, with 
a total of 3 repetitions of all frequency-intensity parameter combinations. Broadband noise bursts of duration 200 ms were presented at $80 \mathrm{~dB}$ SPL, in 100 repetitions with an inter-burst interval of at least $800 \mathrm{~ms}$. (Noise bursts were followed by clicks at $20 \mathrm{~ms}$ and $500 \mathrm{~ms}$ after noise offset, but click-evoked responses are not discussed further here.) Spontaneous neural activity was recorded for 100 intervals of 500 ms without stimulation, in consecutive trial "sweeps" with variable inter-trial intervals dictated by software processing times (Jan Schnupp's BrainWare).

Stimuli were synthesised (TDT RX-6, 195,312.5 Hz sampling rate), attenuated (TDT PA-5), amplified (TDT SA-1), and presented free-field via a loudspeaker (TDT MF-1) positioned at a distance of $15 \mathrm{~cm},-45^{\circ}$ in azimuth to the animal and at the same elevation as the left auditory canal. A sound-attenuating ear plug was placed in the animal's right ear. ABR measurements were made from the left ear (ipsilateral to sound presentation) and IC recordings from the right hemisphere (contralateral to sound presentation). Before each $A B R$ or IC recording experiment, the sound delivery system was calibrated with a $1 / 4$ " microphone (Brüel and Kjær 4939 or GRAS Type 40BF) placed at the approximate position of the animal's ear, to achieve a frequency response that was flat to within $\pm 2 \mathrm{~dB}$ over the full range of sound frequencies tested.

\section{Data analysis}

All $A B R$ and IC recordings and all data analysis procedures were conducted blind to the nature of the injection (AUT00063 or vehicle) and also to whether the animal was noiseexposed or not. More specifically, all in vivo electrophysiology experiments involved two experimenters: one who prepared the injection of AUT00063 solution or vehicle in a syringe wrapped in aluminium foil, and another who performed the in vivo recordings and injection without ever seeing the contents of the syringe. This strategy was necessary to ensure proper blinding during data collection, because the AUT00063 suspension was cloudy while the vehicle solution was clear. Blinding was maintained during data analysis by ensuring that 
estimates of $A B R$ threshold and IC response threshold or frequency tuning were performed for blocks of recorded data files from multiple animals, by an observer blind to the nature of the injection and the noise-exposure status of the animals. Other IC response analyses (e.g. spontaneous rate analysis, noise response analysis) were automated and did not involve subjective judgements.

Statistical tests were non-parametric and performed two-sided with alpha $=0.05$ unless otherwise indicated, with Bonferroni correction for multiple testing applied where appropriate.

ABR data analysis. ABR recordings for each trial were first digitally filtered $(100-3000 \mathrm{~Hz}$, 5th-order Butterworth filter) and then transformed into a differential waveform (difference between electrode signals recorded at the vertex and ipsilateral ear). Differential waveforms for each trial were then averaged and plotted for each sound level as mean +/- s.e.m. across repeated trials. Thresholds were estimated visually (by an experimenter blind to the noise exposure status of the animal) by determining the lowest sound level at which deflections in the ABR waveform were judged to be greater than the background variability (i.e., greater than approximately twice the s.e.m.). Measurements of peak-to-trough wave amplitudes were performed using custom software (Matlab, The Mathworks).

IC data analysis: spontaneous rates. Spontaneous firing rates were calculated for each recording site by taking the median multi-unit firing rate over the 100 repetitions of the 500 ms recording period. We used the median rather than the more conventional mean for this firing rate measure because unlike sound-evoked firing rates, spontaneous firing rates in the IC are close to the "floor" of zero firing rate and are therefore not normally distributed across repeated identical trials. The stability of multi-unit activity at each recording site was evaluated by testing for correlations between sample number and spontaneous rate, with the null hypothesis that there should be no correlation. Multi-units with a significant correlation $(p<0.01)$ were removed from the study. As these unstable recordings occurred primarily in 
three animals (two noise-exposed, one unexposed control), data from those animals were fully removed from the dataset.

Note that for technical reasons, spontaneous activity data were collected in 100 recordings of 500 ms duration; therefore, inter-spike interval (ISI) analysis was not feasible, because the long ISIs typical of spontaneous activity in the IC were not well sampled. We were therefore unable to determine whether changes in spontaneous firing rate following noise exposure or drug injection arose from changes in the incidence of burst firing or in the spontaneous rate during inter-burst intervals. We attempted to address this question using analysis of spikecount Fano factors, and did not find significant effects of noise exposure or drug injection on spontaneous bursting using this measure. However, since variability in Fano factors was high due to the low spontaneous firing rates and short duration of trials used for collecting spontaneous activity data, this analysis was not sufficiently conclusive to be included in the Results.

IC data analysis: tone and noise responses. Multi-unit firing rates evoked by tones of varying frequency and intensity were used to determine the intensity threshold, characteristic frequency (CF, sound frequency evoking a response at intensity threshold) and best frequency (BF, sound frequency evoking maximal response across intensities) for each recording site; these measures were estimated by an experimenter blind to the noise exposure status of the animal and the nature of the injection. Multi-unit firing rates evoked by the broadband noise stimulus were used to determine the magnitude of the noise response at each recording site, which was computed from the mean firing rate across all repeated trials and the entire duration of the broadband noise. Analysis of the effects of AUT00063 on noise responses focuses on noise-evoked firing rates computed using the entire $200 \mathrm{~ms}$ stimulus presentation; results also held when firing rates were instead computed over either onset or sustained response periods alone (e.g., 0-50 ms after noise onset, or last $50 \mathrm{~ms}$ before noise offset). 
IC data analysis: statistical testing. In experiments on the effects of AUT00063 or vehicle injection over time, recordings of spontaneous activity were prioritised and therefore more complete across time points. Interruptions of data acquisition required for anaesthetic checks and animal maintenance were timed to override recordings of tone-evoked and/or noiseevoked responses rather than spontaneous activity when possible. Thus, especially for recordings of evoked responses, sample sizes differed across time points because complete data were collected at only a subset of the possible time points for some of the animals. Moreover, measurements of threshold were coarsely discretised, and spontaneous rate distributions were highly skewed. Therefore data were neither balanced across conditions (as required for repeated-measures ANOVA) nor normally distributed (as required by parametric tests). For these reasons we tested statistical significance at each time-point using the nonparametric Wilcoxon rank-sum test with Bonferroni correction for multiple testing, rather than using a repeated-measures ANOVA. Data are shown in figures as mean \pm s.e.m. for display purposes only. 


\section{Results}

\section{AUT00063 Enhances Kv3 Currents Near Activation Potential}

The effect of AUT00063 on Kv3.1a currents was first investigated using an lonworks ${ }^{\mathrm{TM}}$ automated patch-clamp assay. AUT00063 increased Kv3.1a currents measured at $-15 \mathrm{mV}$ in a concentration-dependent manner (Fig. 1a), with pEC50 values of $5.1 \pm 0.17(7.9 \mu \mathrm{M} ; \mathrm{n}=18)$ for human Kv3.1a currents (Fig. 1b) and comparable results for rat Kv3.1b (Table 1; pEC50 $4.89 \pm 0.02, n=8$ ). The compound showed similar potency to enhance Kv3.2 currents (Table 1). Further tests revealed weaker activity of AUT00063 on Kv3.3 and Kv3.4 channels and little or no activity on a range of other voltage-gated ion channels and pharmacological targets, with a few exceptions (Tables 2 and 3; pXC50 $<4.8$ for all channels and targets except hKv7.1/mink and serotonin 5HT-2A, $-2 \mathrm{C}$ and -3$)$. In the in vivo experiments the compound reached a maximum blood concentration of $6840 \mathrm{ng} / \mathrm{ml}$ (mean maximum value across animals), corresponding to a free concentration of $0.8 \mu \mathrm{M}$ given $95 \%$ protein binding. Thus, pXC50 values well above 5 , which would give a half maximal inhibitory or excitatory concentration of $<10 \mu \mathrm{M}$, would be considered relevant to in vivo interaction.

The effect of AUT00063 on the voltage dependence of activation of hKv3.1a currents was studied using whole-cell patch clamp recording using the QPatch automated patch-clamp platform. Kv3.1 currents were evoked by depolarizing the membrane from a holding potential of $-90 \mathrm{mV}$ to test potentials from $-60 \mathrm{mV}$ to $+30 \mathrm{mV}$ in $10 \mathrm{mV}$ increments in the presence and in the absence of AUT00063. The addition of AUT00063 increased KV3.1 currents at voltages close to the threshold for $\mathrm{Kv} 3$ channel activation ( -40 to $-0 \mathrm{mV}$ ) whereas little or no potentiation of $\mathrm{Kv} 3$ currents was observed at higher voltages $(>+20 \mathrm{mV})$. When the normalised conductance was plotted as a function of membrane voltage, there was a leftward shift in the voltage dependence of activation to more negative potentials after addition of the compound (Fig. 1c). The half-activation potential ( $\mathrm{V} 1 / 2$ max) was calculated by fitting the voltage curves to a single Boltzmann isotherm. The $\mathrm{V}^{1} / 2 \max$ was $-9.9 \pm 0.8 \mathrm{mV}$ 
$(n=7)$ in control, $-12.3 \pm 0.4 \mathrm{mV}(\mathrm{n}=7)$, and $-18.9 \pm 0.7 \mathrm{mV}(\mathrm{n}=6)$ after addition of AUT00063 at $10 \mu \mathrm{M}$ and $30 \mu \mathrm{M}$, respectively. The slope (k) of the fitted curves was $13.5 \pm 0.79 \quad(n=7)$ in control animals and $12.91 \pm 0.37(n=7)$ and $13.60 \pm 0.66 \quad(n=6)$ after addition of AUT00063 at $10 \mu \mathrm{M}$ and $30 \mu \mathrm{M}$, respectively.

Additionally, the effects of AUT00063 on channel deactivation kinetics were investigated on tail currents measured at $-40 \mathrm{mV}$ (Fig. $1 \mathrm{~d}$, inset) in the lonWorks ${ }^{\mathrm{TM}}$ assay. The rate of deactivation was slowed in a concentration-dependent manner (Fig. 1d).

\section{AUT00063 Blood Levels Rise Rapidly After Injection and Are Maintained for Two Hours}

Pharmacokinetic investigations in 4 male CBA/Ca mice were designed to mimic the dosage conditions used in in vivo electrophysiology experiments. Following i.p. injection of $90 \mathrm{mg} / \mathrm{kg}$ of AUT00063, blood concentrations of the drug were assessed at regular intervals for up to 2 hours. Data revealed a rapid rise in blood concentrations of AUT00063 within the first 30 minutes after dosing, with relatively stable levels maintained for up to $120 \mathrm{~min}$ (Fig. 2). In all 4 mice, blood concentrations exceeded $80 \%$ of maximum by 30 min after the injection. Maximum blood concentrations of AUT00063 for the 4 mice were 6570, 7070, 7860 and $5850 \mathrm{ng} / \mathrm{ml}$ (mean +/- s.d., $6840+/-846 \mathrm{ng} / \mathrm{ml}$ ), and were reached at 120, 30, 60 and 120 min (median 90 min) after i.p. injection, respectively. These blood levels of AUT00063 were similar to those observed in other pharmacokinetic studies performed in different mouse models with a lower dose of $60 \mathrm{mg} / \mathrm{kg}$ i.p. (data not shown). Solubility of the AUT00063 compound is approximately $1.2 \mathrm{mg} / \mathrm{ml}$ in 12.5 captisol/HPMC/Tween80; the volume of administration is $10 \mathrm{ml} / \mathrm{kg}$, therefore the absorption after IP administration may be limited by the fraction of the dose in solution.

\section{Octave-Band Noise Exposure Produces Frequency-Specific ABR Threshold Shifts}

To test the effects of AUT00063 on auditory midbrain activity in an animal model of hearing loss following noise exposure, we performed electrophysiological studies using an additional 
21 male CBA/Ca mice divided into four experimental groups: noise-exposed animals that received AUT00063, noise-exposed animals that received inert vehicle, unexposed control animals that received AUT00063, and unexposed control animals that received inert vehicle. Noise exposure involved presentation of an octave-band noise (8-16 kHz) at $105 \mathrm{~dB}$ SPL for 2 hours under anaesthesia.

In animals exposed to noise, ABR recordings 1 day after noise exposure revealed significant threshold shifts at 11, 16, 32 and $48 \mathrm{kHz}$ (Fig. 3a, light grey line versus black line; Wilcoxon signed-rank test, $\mathrm{p}<0.005$ at 11, 16, 32 and $48 \mathrm{kHz}$ ). Four weeks after noise exposure, hearing thresholds had recovered to pre-exposure values at 32 and $48 \mathrm{kHz}$ (Fig. 3a, dark grey line versus black line; Wilcoxon signed-rank test, $p>0.2$ at 32 and $48 \mathrm{kHz}$ ) but remained elevated at 11 and $16 \mathrm{kHz}(p=0.02$ at $11 \mathrm{kHz}$ and $\mathrm{p}=0.006$ at $16 \mathrm{kHz})$. Moreover, the amplitudes of ABR wave I in click-evoked responses (Fig. 3b) were significantly reduced relative to pre-exposure values (ANOVA, $\mathrm{p}<0.01$ ), and the slope of the growth of wave I with sound intensity was shallower. These findings are suggestive of noise-induced cochlear damage, possibly including deafferentation of auditory nerve fibres (Kujawa and Liberman, 2009), a widely reported consequence of noise exposure and likely cause of "hidden" hearing loss (Schaette and McAlpine, 2011).

\section{Noise Exposure Produces CF-Specific Shifts in Tone Response Thresholds of IC}

\section{Neurons}

Recordings of spontaneous and evoked multi-unit activity in the inferior colliculus were obtained from 21 male CBA/Ca mice under terminal anaesthesia, before and after a single injection of either AUT00063 or vehicle. Data from 18 animals has been included in the population analysis; all data from the remaining three animals were excluded due to instability in some of the recordings in those animals (see Methods - In Vivo Electrophysiology: Data analysis for details). The remaining dataset consisted of recordings from 10 noise-exposed animals (5 used for AUT00063 injection experiments, 5 vehicle) and 
8 control animals (3 AUT00063, 5 vehicle). We focused first on comparing IC characteristics between noise-exposed mice and unexposed control animals, by analysing spontaneous and tone-evoked activity recorded before AUT00063 or vehicle injections.

Tone response thresholds for multi-unit activity recorded in the IC of individual control (unexposed) animals ranged from 0-50 dB SPL, with a median of $10 \mathrm{~dB}$ SPL. At approximately $70 \%$ of recording sites in control animals, the tone response threshold was $\leq$ $15 \mathrm{~dB}$ SPL. The range of tone response thresholds was similar in noise-exposed mice (0-60 dB SPL); however, the median threshold of $20 \mathrm{~dB}$ SPL was significantly higher in noiseexposed than control animals (Wilcoxon rank-sum test, $p=3 \times 10^{-9}$ ), indicating hearing loss. When recordings were grouped according to characteristic frequency, tone response thresholds were observed to be significantly higher in noise-exposed than control animals for multi-units with CFs between 11 and $16 \mathrm{kHz}$ (Fig. 3c; Wilcoxon rank-sum test, $p=1 \times 10^{-5}$ ). Thus, tone response thresholds in noise-exposed animals were significantly and specifically elevated at multi-unit recording sites with frequency tuning within the frequency range of the 8-16 kHz octave-band noise used for noise exposure.

The range of characteristic frequencies of IC units from control animals covered the full hearing range of the mouse, with the greatest proportion of units tuned around $24 \mathrm{kHz}$ (Fig. $3 d$, blue line). In contrast, the distribution of CFs in the noise-exposed animals was significantly different (Kolmogorov-Smirnov test, $p=0.0001$ ), with a peak in the distribution around $12 \mathrm{kHz}$ (Fig. 3d, red line), i.e. in the middle of the frequency range of the octave-band noise used for the noise exposure. Similar results were obtained for best frequencies (data not shown). These findings demonstrate that while noise exposure produced significantly elevated tone response thresholds within the frequency range of noise exposure (Fig. 3c), the likelihood of observing tuned tone-evoked multi-unit activity within this frequency range was nevertheless increased rather than reduced (Fig. 3d). 


\section{Noise Exposure Increases Spontaneous Activity in IC Neurons with CFs Near Exposure Frequencies}

Spontaneous firing rates were generally low in control animals $(1.2 \pm 0.25$ spikes $/ \mathrm{sec})$. In the noise-exposed group, spontaneous firing rates of IC neurons were significantly higher (2.3 \pm 0.28 spikes/sec; Wilcoxon rank-sum test for difference in medians, $p=0.0002)$. Increases in spontaneous firing rates were restricted to neurons with characteristic frequencies $<22 \mathrm{kHz}$, i.e. within and up to approximately $1 / 2$ octave above the noise-exposure frequency range (Fig. 3e; Wilcoxon rank-sum test, $p=0.004$ for $11-16 \mathrm{kHz}$ ). Thus, spontaneous firing rates in multiunit IC recordings were significantly higher in noise-exposed than control animals, specifically at recording sites with CFs near the frequency range of the octave-band noise used for noise exposure.

\section{AUT00063 Counteracts CF-specific Increases in IC Spontaneous Rates Following Noise Exposure}

Spontaneous activity, frequency-intensity response areas and responses to noise bursts were measured before, immediately after, and at 30-minute intervals for up to 2 hours after i.p. injection of AUT00063 at $90 \mathrm{mg} / \mathrm{kg}$ or vehicle (Fig. 4a). All recordings included measurements at the pre-injection time point and at least two post-injection time points; for n's at each time point, see Fig. 4 legend. We tested for a difference between AUT00063 and vehicle effects at each time point after injection using the non-parametric Wilcoxon rank-sum test for difference in medians, with Bonferroni correction for multiple testing across the six time points. (We used this approach rather than a repeated-measures ANOVA because the data were neither balanced across conditions nor reliably normally distributed; see Methods - In Vivo Electrophysiology: Data analysis.)

To analyse the effects of AUT00063 versus vehicle injection on spontaneous activity in the IC, we divided IC multi-units into two sets according to the pre-injection CFs. The first set consisted of multi-unit recordings with $\mathrm{CF}<22 \mathrm{kHz}$, i.e. within the $\mathrm{CF}$ range in which we had 
observed increases in spontaneous firing rates following noise exposure (Fig. 3e).

Recordings with $\mathrm{CF} \geq 22 \mathrm{kHz}$ formed the second set. In this frequency range, spontaneous firing rates were unchanged after noise exposure (Fig. 3e). Data are shown in Fig. 4 as mean \pm s.e.m. for display purposes; however, all statistical tests were non-parametric, as appropriate given that spontaneous rates were not normally distributed.

In noise-exposed animals, AUT00063 injection decreased spontaneous activity and reduced spontaneous firing rates to control levels in multi-unit recordings with $\mathrm{CF}<22 \mathrm{kHz}$, whereas spontaneous firing rates remained elevated after vehicle injection (Fig. $4 \mathrm{~b}$ and c, top panels). This effect of AUT00063 injection was clearly evident whether we analysed absolute spontaneous firing rates (Fig. 4b) or changes in spontaneous firing rate from the pre-injection baseline for each recording (Fig. 4c). The difference in absolute levels of spontaneous activity between the AUT00063 group and the vehicle group was significant at all time points following the injection (Fig. 4b; Wilcoxon rank-sum tests, all post-injection time points $p<$ 0.0001). The difference between AUT00063 and vehicle groups was also significant for changes in spontaneous firing rate relative to pre-injection baseline at all but the last postinjection time point (Fig. 4c; Wilcoxon rank-sum tests, $(0,20]$, [30,50], $[60,80]$ and $[90,110]$ min time points all $p<0.005)$.

In contrast, for multi-unit recordings with $\mathrm{CF} \geq 22 \mathrm{kHz}$ in noise-exposed animals, no significant differences were observed between the effects of AUT00063 and vehicle injection on either absolute levels of spontaneous activity (Fig. 4d, top panel; Wilcoxon rank-sum tests, n.s. for all time points) or changes in spontaneous firing rate relative to pre-injection baseline (Fig. 4e, top panel; n.s. for all time points). Moreover, in unexposed control animals, there were minimal differences between the effects of AUT00063 and vehicle on spontaneous activity in recordings with either CF $<22 \mathrm{kHz}$ or $\mathrm{CF} \geq 22 \mathrm{kHz}$ (Fig. 4b-e, bottom panels). In fact, the only significant difference between the effects of AUT00063 and vehicle injection observed in unexposed control animals was that in recordings with $\mathrm{CF}<22 \mathrm{kHz}$, 
spontaneous rate change relative to pre-injection baseline was higher following AUT00063 than vehicle injection at the $[60,80]$ min time point (Fig. 4c, bottom panel; Wilcoxon rank-sum tests, $p=0.002$ ). Thus, injection of AUT00063 lowered IC spontaneous firing rates only in noise-exposed animals, and only in recordings with CFs in the range where pathological increases in spontaneous firing rates were observed following noise exposure.

\section{No Significant Effects of AUT00063 on Frequency Tuning or Thresholds of Tone- Evoked Responses in IC}

We also tested the effects of AUT00063 versus vehicle injections on frequency tuning and thresholds of evoked responses to tones. For these investigations, we focused on analysis of changes in CF or threshold relative to the pre-injection baseline rather than absolute measures, since measures of change relative to baseline are less sensitive to the expected variability in CF and threshold measures across different recording sites. Similar results were obtained in all cases for analysis of absolute CFs and thresholds. Sampling of time points was less complete for CF and threshold data than for spontaneous rate data (see Methods In Vivo Electrophysiology: Data analysis). However, all recordings used for this analysis included a pre-injection measurement and at least one post-injection measurement (see Fig. 5 legend for n's at each time point).

Relative to vehicle injection, AUT00063 injection had no significant effect on characteristic frequencies (Fig. 5a), best frequencies (data not shown), or thresholds (Fig. 5b) of toneevoked responses in either noise-exposed or control animals (Wilcoxon rank-sum tests, n.s. at all time points). Similar results were obtained when analysis was performed separately on recordings with $\mathrm{CF}<22$ and $\mathrm{CF} \geq 22 \mathrm{kHz}$ (data not shown; Wilcoxon rank-sum tests, n.s. at all time points in both groups). Thus, there was no significant effect of AUT00063 on frequency tuning or thresholds of tone-evoked responses in IC, in either noise-exposed animals or unexposed control animals. 
To examine the effects of AUT00063 on the magnitude of evoked activity in IC, we recorded multi-unit responses to repeated presentations of a $200 \mathrm{~ms}, 80 \mathrm{~dB}$ SPL broadband noise (Fig. 6). This stimulus typically elicited strong responses at multiple simultaneously recorded sites along the multi-electrode probe. Noise-evoked responses were not collected at all time points in all recordings (see Methods - In Vivo Electrophysiology: Data analysis); however, all recordings included a pre-injection measurement and at least one post-injection measurement (see Fig. 6 legend for n's at each time point). To reduce the impact of variability in evoked response characteristics across different recording sites, we focused on analysis of changes in noise response magnitude relative to the pre-injection baseline, but consistent results were also obtained for absolute response magnitudes.

AUT00063 significantly reduced evoked activity at a few post-injection time points in noiseexposed animals (Fig. 6), both for recordings with $\mathrm{CF}<22 \mathrm{kHz}$ (Fig. 6a; Wilcoxon rank-sum tests, time points $[30,50] \min , p=0.001$ and $[60,80]$ min, $p=0.008)$ and for recordings with $\mathrm{CF} \geq 22 \mathrm{kHz}$ (Fig. 6b; time points $(0,20] \mathrm{min}, \mathrm{p}=0.0001$ and $[60,80] \mathrm{min}, \mathrm{p}=0.006$ ). These effects of AUT00063 on evoked responses in noise-exposed animals did not depend on the evoked response period; similar results were obtained when evoked firing rates were calculated across the entire 200 ms stimulus duration as in Fig. 6, or only for the first 50 ms $(\mathrm{CF}<22 \mathrm{kHz}:[30,50] \min , \mathrm{p}=0.001 ; \mathrm{CF} \geq 22 \mathrm{kHz}:(0,20] \min , \mathrm{p}=0.0001$ and $[60,80] \mathrm{min}, \mathrm{p}$ $=0.006)$ or last $50 \mathrm{~ms}(\mathrm{CF}<22 \mathrm{kHz}:[30,50] \mathrm{min}, \mathrm{p}=0.002$ and $[60,80] \mathrm{min}, \mathrm{p}=0.003 ; \mathrm{CF} \geq$ $22 \mathrm{kHz}:(0,20] \min , \mathrm{p}=0.008)$ of the noise stimulus. Results for unexposed control animals were somewhat inconsistent, with no significant differences between effects of AUT00063 and vehicle injections in some analyses (e.g., evoked firing rate relative to pre-injection baseline, calculated either over the entire 200 ms stimulus interval as in Fig. 6 or for the last 50 ms of the noise stimulus) but significant differences at a few post-injection time points in other analyses (e.g., evoked firing rate in first 50 ms of noise stimulus, time point $[120,140]$ $\min \mathrm{p}=0.008$ in both $\mathrm{CF}<22 \mathrm{kHz}$ and $\mathrm{CF} \geq 22 \mathrm{kHz}$ recordings; data not shown). Thus, there is some evidence that AUT00063 reduces evoked firing rates in the IC. However, unlike the 
suppressive effect of AUT00063 on spontaneous firing rates, the effect of AUT00063 on evoked firing rates was not specific to IC recordings with CFs in the frequency range most affected by noise exposure, nor specific to noise-exposed animals. 


\section{Discussion}

Here we have studied the effect of AUT00063, a modulator of Kv3 potassium channels, on spontaneous as well as evoked neural activity in the inferior colliculus of noise-exposed and control mice. Octave-band noise exposure produced elevated levels of spontaneous activity in the IC at multi-unit recording sites with characteristic frequencies within or just above the frequency band used for noise exposure. In noise-exposed mice, administration of AUT00063 reduced IC spontaneous activity to levels typical of control animals, specifically at recording sites with CFs in the frequency range associated with elevated spontaneous activity following noise exposure. There was no significant effect of AUT00063 on spontaneous firing rates at recording sites with CFs outside this frequency range in noiseexposed animals, and no suppressive effect of AUT00063 on spontaneous firing rates in unexposed control mice. Moreover, there was no significant effect of AUT00063 on thresholds or frequency tuning of tone-evoked responses in either noise-exposed or unexposed control mice. Noise-evoked response magnitudes were reduced at a few time points following AUT00063 injection compared to vehicle injection, but these suppressive effects on evoked activity were not specific to recording sites with CFs in the frequency range most affected by noise exposure. In contrast, AUT00063 selectively re-normalised pathologically increased spontaneous activity in the IC of noise-exposed mice.

The noise exposure used in our study caused an increase in hearing thresholds and a reduction in the amplitude of $A B R$ wave I as well as the slope of its growth function. Increases in spontaneous firing rates in the IC were observed for neurons tuned to sound frequencies within and slightly above the frequency range of noise exposure, which also matched the region of hearing loss. A similar relationship between hearing threshold increase and neuronal hyperactivity has previously been observed in the IC of guinea pigs (Coomber et al., 2014; Mulders and Robertson, 2009; Mulders et al., 2011). 
Our results are consistent with the hypothesis that down-regulation of Kv3 currents in the auditory brainstem after noise exposure (Pilati et al., 2012) could contribute to the generation of central auditory hyperactivity, and that AUT00063, which lowers the activation potential for Kv3.1 currents, might counteract this process. Down-regulation of Kv3 currents following noise exposure was previously observed in fusiform cells of the dorsal cochlear nucleus (Pilati et al., 2012). It is well known that fusiform cell populations also represent the major source of DCN hyperactivity observed after noise exposure (Brozoski et al., 2002; Shore et al., 2008; Finlayson and Kaltenbach, 2009; Wu et al., 2016). The hyperactive state of DCN fusiform cells could be associated with the hyperactivity observed in the IC following noise exposure, since the IC receives a direct projection from DCN fusiform cells. Thus it is possible that in the present study, AUT00063 enhanced the activation of Kv3.1 currents in fusiform cells at more negative membrane potentials, leading to reduction in spontaneous activity in fusiform cells and consequently in the IC.

In support of this possible mechanism, Glait et al. (2018) recently observed that AUT00063 reduced spontaneous activity of DCN fusiform cells in noise-exposed hamsters. The same study also found that AUT00063 had a suppressive effect on spontaneous activity of DCN fusiform cells in control (unexposed) animals, a result which contrasts with our finding that AUT00063 had no significant effect on spontaneous activity of IC neurons in unexposed animals. This difference in results may well reflect differences between DCN and IC, or between hamsters and mice. However, previous work has shown that ablation of the DCN reduced IC hyperactivity in noise-exposed hamsters whereas it did not affect spontaneous IC activity in control hamsters (Manzoor et al., 2012). Therefore, it is possible that AUT00063induced changes in DCN activity might reduce pathological hyperactivity in the IC of noiseexposed animals, but not normal baseline spontaneous rates in the IC of unexposed animals. 
Our IC recordings were performed four weeks after noise exposure in noise-exposed animals and unexposed control animals. In guinea pigs four weeks after noise exposure, auditory midbrain hyperactivity depends on input from the cochlea (Mulders and Robertson, 2009). Therefore, amplification of spontaneous auditory nerve activity may also play a role in generating IC hyperactivity following noise exposure. The exact location of the amplification mechanism, and how Kv3 channels might be involved, remain to be determined. Further studies incorporating a sham-exposure control group would be necessary to address questions about the mechanisms underlying observed effects of noise exposure. Here, our primary focus was instead on comparing the effects of AUT00063 versus vehicle administration, either in noise-exposed animals or in unexposed controls.

Reduction of spontaneous firing rates in the IC occurred rapidly after AUT00063 administration, consistent with the rapid elevation in blood levels of the drug observed following i.p. injection in mice and suggesting rapid access of the drug into the central nervous system. Moreover, both changes in IC spontaneous firing rates and elevated blood levels of the drug were maintained over the two-hour duration of experiments. Thus, the overall time course for effects of AUT00063 injection on IC spontaneous firing rates was consistent with the results of the pharmacokinetics study. Notably, effects of AUT00063 on the magnitude of noise-evoked activity were much less consistent in time course, as well as less specific to recording sites tuned near the frequency range used for noise trauma.

The pharmacological studies of AUT00063 shown here (Fig. 1) were carried out on hKv3.1a. The Kv3.1 gene has two splice variants, Kv3.1a and Kv3.1b, differing at only the level of their intracellular C terminus (Kv3.1a is the shorter isoform). The temporal expression of these two splice variants differs during development; Kv3.1a (also called Kv3.1ß) is the predominant transcript in developing neurons whereas Kv3.1b (also called Kv3.1d) predominates in adult neurons (Perney et al., 1992). An intriguing possibility is that both isoforms form heteroligomers with each other which change in composition not only during development 
(Kv3.1 channel composed mostly of Kv3.1a subunits early in development but Kv3.1b subunits later in development) but also following stimulation such as noise exposure (Leão et al., 2010; Si-qiong et al., 1998). Since the two isoforms differ only in their intracellular C terminus, and AUT00063 most likely binds to the extracellular portion of the channel, we would expect the compound to affect both isoforms similarly. Consistent with this expectation, the compound was tested on rKv3.1b channels (Brown et al, 2016, plus data not shown) and displayed similar effects as observed on hKv3.1a channels.

However, we cannot exclude the possibility that the observed results of AUT00063 administration in vivo arose from other effects of the compound on mouse Kv3.1b channels, or were mediated by interactions with targets other than Kv3 channels (cf. Lobarinas et al., 2011; Li et al., 2013; Hurley and Sullivan, 2012). The dosage of AUT00063 used in these experiments $(90 \mathrm{mg} / \mathrm{kg}$ ) was relatively high compared to dosages used in other in vivo studies of AUT00063 (typically $30 \mathrm{mg} / \mathrm{kg}$ or $60 \mathrm{mg} / \mathrm{kg}$; personal communication, Autifony Therapeutics Limited; Glait et al., 2018), although blood levels of the drug measured here were similar to those observed previously with $60 \mathrm{mg} / \mathrm{kg}$ dosage in other mouse models. We did not evaluate the effects of lower dosages of AUT00063 on auditory midbrain activity. Notably, however, the observed effects of $90 \mathrm{mg} / \mathrm{kg}$ of AUT00063 were quite specific. Significant effects of AUT00063 on IC spontaneous activity were observed only in noiseexposed, not control, mice, and primarily at recording sites with CFs near the frequencies of previous noise exposure. Moreover, there was no significant effect of AUT00063 on thresholds or frequency tuning of tone-evoked responses. Therefore, it seems unlikely that these effects arose from non-specific interactions of high doses of AUT00063 with targets for which the compound has low selectivity. Similarly, while we cannot rule out the possibility that the observed effects arose from some interaction of AUT00063 with the anaesthetics used in this study, the lack of any significant effects of AUT00063 on IC responses in anaesthetised control animals makes this explanation seem unlikely. 
We conclude that AUT00063, a modulator of Kv3 channels, counteracts elevation of spontaneous firing rates in the auditory midbrain following octave-band noise exposure. These findings raise the possibility that AUT00063 could be used to normalise central auditory pathologies arising from noise exposure in humans.

\section{Acknowledgements}

This study was funded by Autifony Therapeutics Limited. R.S. was supported by the British Tinnitus Association. 


\section{Tables}

Table 1. Selectivity of AUT00063 versus Kv3 channels. Data shown are the mean determinations from at least 2 independent lonworks ${ }^{\circledR}$ patch-clamp assays.

\begin{tabular}{|l|l|}
\hline Channel & Results \\
\hline hKv3.1 & pEC50 $5.1 \pm 0.17(n=18)$ \\
\hline rKv3.1 & pEC50 4.89 $\pm 0.02(n=8)$ \\
\hline hKv3.2 & pEC50 5.1 $\pm 0.20(n=18)$ \\
\hline hKv3.3 & pEC50 $4.8(n=4)$ \\
\hline hKv3.4 & plC50 $4.7(n=4)$ \\
\hline
\end{tabular}

Table 2. Selectivity of AUT00063 versus other voltage-gated ion channels. Data are mean data obtained from functional electrophysiology assays carried out on behalf of Autifony by Scinovo (GSK), UK.

\begin{tabular}{|c|c|c|}
\hline Channel & Results & Assay \\
\hline hKv1.5 & plC50 <4.3 $(n=2)$ & Ionworks ${ }^{\circledR}$ patch-clamp \\
\hline hKv7.1/mink & plC50 $5.0(n=4)$ & Ionworks $\AA$ patch-clamp \\
\hline hERG & plC50 <4.5 $(n=4)$ & Patch-Xpress $₫$ patch-clamp \\
\hline hNav1.5 & plC50 $4.1(n=2)$ & Ionworks $®$ patch-clamp \\
\hline hCav1.2 & $\mathrm{plC50}<4.8(\mathrm{n}=2)$ & Ionworks $₫$ patch-clamp \\
\hline
\end{tabular}


Table 3. Selectivity of AUT00063 versus other pharmacological targets. In each experiment, the respective reference compound was tested concurrently with AUT00063. Data are the mean pXC50 (pIC50 for antagonist or pEC50 for agonist) values from ligand binding assays conducted on behalf of Autifony by CEREP (France).

\begin{tabular}{|l|l|c|c|c|}
\hline Assay & $\begin{array}{l}\text { Assay } \\
\text { Mode }\end{array}$ & Modifier & pXC50 & $\mathbf{n}$ \\
\hline Adenosine 2a & Ag & $<$ & 4.5 & 3 \\
\hline Adrenergic Alpha-1b & Ant & $<$ & 4.4 & 3 \\
\hline Adrenergic Alpha-2c & $\mathrm{Ag}$ & $<$ & 4.5 & 2 \\
\hline Adrenergic Beta-2 & $\mathrm{Ag}$ & $<$ & 4.5 & 2 \\
\hline Adrenergic Beta-2 & $\mathrm{Ant}$ & $<$ & 4.5 & 2 \\
\hline Alpha-1 nicotinic AChR & Open & $<$ & 4.3 & 2 \\
\hline Cannabinoid B2 & $\mathrm{Ag}$ & $<$ & 4.5 & 2 \\
\hline Dopamine D1 & $\mathrm{Ant}$ & $<$ & 4.5 & 2 \\
\hline Dopamine D2 & $\mathrm{Ag}$ & $<$ & 4 & 2 \\
\hline Dopamine D2 & $\mathrm{Ant}$ & $<$ & 4 & 2 \\
\hline GSK3b & $\mathrm{Ant}$ & $<$ & 4.5 & 1 \\
\hline Histamine H1 & $\mathrm{Ant}$ & $<$ & 4.4 & 2 \\
\hline LCK & $\mathrm{Ant}$ & $<$ & 4.5 & 1 \\
\hline Muscarinic M1 & $\mathrm{Ag}$ & $<$ & 4.8 & 2 \\
\hline Muscarinic M1 & $\mathrm{Ant}$ & $<$ & 4.8 & 2 \\
\hline Muscarinic M2 & $\mathrm{Ag}$ & $<$ & 4.8 & 2 \\
\hline Muscarinic M2 & $\mathrm{Ant}$ & $<$ & 4.8 & 2 \\
\hline Neurokinin NK1 & $\mathrm{Ant}$ & $<$ & 4.4 & 2 \\
\hline Mu Opioid & $\mathrm{Ag}$ & $<$ & 4 & 2 \\
\hline Mu Opioid & $\mathrm{Ant}$ & $<$ & 4 & 2 \\
\hline Kappa Opioid & $\mathrm{Ag}$ & $<$ & 4 & 2 \\
\hline Kappa Opioid & $\mathrm{Ant}$ & $<$ & 4 & 2 \\
\hline PDE4B & $\mathrm{Ant}$ & $=$ & 4.4 & 1 \\
\hline PI3K\&gamma & $\mathrm{Ant}$ & $<$ & 4.5 & 1 \\
\hline Sertonin 5HT-1B & $\mathrm{Ag}$ & $<$ & 4.5 & 2 \\
\hline Serotonin 5HT-1B & $\mathrm{Ant}$ & $<$ & 4.5 & 2 \\
\hline Serotonin 5HT-2A & $\mathrm{Ag}$ & $<$ & 4.4 & 4 \\
\hline Serotonin 5HT-2A & $\mathrm{Ant}$ & $=$ & 5.9 & 2 \\
\hline Serotonin 5HT-2C & $\mathrm{Ag}$ & $<$ & 4.4 & 2 \\
\hline Serotonin 5HT-2C & $\mathrm{Ant}$ & $=$ & 4.8 & 2 \\
\hline Serotonin 5HT-3 & Open & $<$ & 4.3 & 2 \\
\hline Serotonin 5HT-3 & $\mathrm{Block}$ & $=$ & 4.9 & 2 \\
\hline COX2 & $\mathrm{Block}$ & $<$ & 4 & 2 \\
\hline Noradrenaline transporter & $\mathrm{Ant}$ & $<$ & 4 & 3 \\
\hline Serotonin transporter & $\mathrm{Ant}$ & $<$ & 4 & 2 \\
\hline & & & & \\
\hline
\end{tabular}




\section{Figures}

Figure 1. In vitro pharmacological characterisation of AUT00063 on hKv3.1 mediated currents. a) Representative traces of human Kv3.1a mediated currents. Currents were evoked by voltage steps to $-15 \mathrm{mV}$ before and after addition of three concentrations of AUT00063 (1.5, 12.5 and $25 \mu \mathrm{M})$. b) Responses normalised to a positive pharmacological standard were averaged and plotted against AUT00063 concentration. Estimated pEC50 and maximum response are indicated on the graph. c) The effects of AUT00063 on the activation voltage-dependence were evaluated by plotting normalised conductance as a function of membrane voltages. AUT00063 produced a significant, concentration-dependent shift of the activation of Kv3.1a channels to more hyperpolarised potentials (unpaired $t$-test for difference in $\mathrm{V}^{1} \frac{1}{2}$ (see text): $\mathrm{p}=0.03$ for AUT00063 $10 \mu \mathrm{M}$ versus $0.1 \%$ DMSO vehicle, $\mathrm{p}=0.0008$ for AUT00063 $30 \mu \mathrm{M}$ versus vehicle). AUT00063 did not affect the slope $\mathrm{k}$ (unpaired $t$-test, see text for k values: $p=0.79$ for AUT00063 $10 \mu \mathrm{M}$ versus $0.1 \%$ DMSO vehicle, $p=0.68$ for AUT00063 $30 \mu \mathrm{M}$ versus vehicle). d) Plot of the mean change in rate of deactivation of Kv3.1 current following addition of AUT00063. Inset shows a representative recording of tail currents (arrow) before and after application of AUT00063 $12.5 \mu \mathrm{M}$. Tail currents were recorded at $-40 \mathrm{mV}$ following a $50 \mathrm{msec}$ pulse at $+40 \mathrm{mV}$. AUT00063 slows the rate of deactivation of Kv3.1 currents.

Figure 2. Time course of elevation in blood concentration of AUT00063 following i.p. drug injection in mice. Different symbols and line colours indicate each of 4 different CBA/Ca mice used for this test. Symbols mark individual blood concentrations of AUT00063 measured at different time points for each mouse. 
Figure 3. Effects of noise exposure on central auditory responses in the mouse. a) Thresholds for tone-evoked auditory brainstem responses before (black line) and 1 day (light grey line) as well as 4 weeks (red line) after noise exposure, displayed as mean \pm s.e.m. across animals ( $n=10$ animals, 1 ear tested per animal). Thresholds were significantly increased at $11-48 \mathrm{kHz}$ on day 1 , and at 11 and $16 \mathrm{kHz} 4$ weeks following noise exposure. b) Amplitude of click-evoked ABR wave I as a function of sound level (mean \pm s.e.m. across animals). Here and in c-e, red indicates noise-exposed mice tested 4 weeks after exposure $(n=10)$, and blue indicates unexposed control mice $(n=8)$. In noise-exposed mice, there was not only a threshold shift, but also a decrease in the slope of the growth of ABR wave I amplitude with sound level. c) Thresholds for responses of IC multi-units to tones as a function of characteristic frequency, displayed as the mean \pm s.e.m. across animals of the mean threshold within each CF band for each animal (calculated from a total of 100 recordings in 10 noise-exposed mice and 86 recordings in 8 unexposed control mice). Thresholds were significantly increased for units with CF 11-16 kHz (Wilcoxon rank-sum test). d) Distribution of characteristic frequencies of IC multi-units. Plots show mean \pm s.e.m. across animals of the percentage of cells with each CF for each animal; number of recordings and mice as in c. The CF distributions differed significantly between noiseexposed and unexposed control animals (Kolmogorov-Smirnov test, $p=0.0001$ ), with a higher percentage of CFs around $12 \mathrm{kHz}$ and lower percentage around $24 \mathrm{kHz}$ in noiseexposed animals than in unexposed controls (Wilcoxon rank-sum tests). e) Spontaneous firing rates of IC multi-units grouped by characteristic frequency; plotting conventions and number of recordings and mice as in $\mathbf{c}$ and $\mathbf{d}$. Spontaneous firing rates were significantly increased for multi-unit recordings with CF 11-16 kHz (Wilcoxon rank-sum test).

Figure 4. AUT00063 normalises pathological increases in spontaneous firing rates in the IC four weeks after noise exposure. a) Schematic depiction of experiment sequence. b) 
Absolute spontaneous firing rates (mean \pm s.e.m. across recordings) for multi-units with characteristic frequencies $(\mathrm{CFs})<22 \mathrm{kHz}$, i.e. within the frequency range in which spontaneous firing rates were significantly elevated following noise exposure. Magenta, AUT00063 injection; green, vehicle injection. Grey bands indicate mean spontaneous rate \pm s.e.m. across recordings obtained in the unexposed AUT00063 group prior to injection. Asterisks mark significant differences in Wilcoxon rank-sum tests between AUT00063 and vehicle injection groups at each time point, with Bonferroni correction for multiple testing across time points. (Top) In recordings with $\mathrm{CF}<22 \mathrm{kHz}$ from noise-exposed animals, AUT00063 reduced spontaneous rates to the levels observed in unexposed animals. (Bottom) In unexposed animals, AUT00063 had no effect on spontaneous firing rates. c) Same analysis but for changes in spontaneous rate relative to the pre-injection baseline for each recording. d) For multi-units with $\mathrm{CF} \geq 22 \mathrm{kHz}$, there were no significant differences in absolute spontaneous firing rates between AUT00063 and vehicle groups, either in exposed or unexposed animals. e) Same analysis but for changes in spontaneous rate relative to the pre-injection baseline for each recording. In each plot, $\mathrm{n}$ indicates the number of multi-unit recordings for the pre-injection time point; all recordings included the pre-injection time point and at least two post-injection time points. For $\mathrm{CF}<22 \mathrm{kHz}$, values of $\mathrm{n}$ at each of the six time points were: noise-exposed, vehicle injection, $n=30,30,30,27,27,19$; noise-exposed, AUT00063 injection, $n=37,37,37,27,24,33$; unexposed, vehicle injection, $n=23,23,23$, 17, 12, 13; unexposed, AUT00063 injection, $\mathrm{n}=21,21,21,21,21,12$. For $\mathrm{CF} \geq 22 \mathrm{kHz}$, values of $n$ at each of the six time points were: noise-exposed, vehicle injection, $n=17,17$, 17, 13, 13, 11; noise-exposed, AUT00063 injection, $\mathrm{n}=16,16,16,16,9$, 9; unexposed, vehicle injection, $n=19,19,19,8,8,8$; unexposed, AUT00063 injection, $n=23,23,23,23$, 23,17 . 
Figure 5. No differences between effects of AUT00063 and vehicle on IC multi-unit CFs and thresholds. All plots show mean \pm s.e.m. change from pre-injection baseline for each recording, for experiments involving injection of either AUT00063 (magenta) or vehicle (green) in noise-exposed or unexposed animals. There were no significant differences in effects of AUT00063 versus vehicle on either CFs (a) or tone response thresholds (b) at any of the time points (Wilcoxon rank-sum tests, all n.s.), whether results were analysed for pooled recordings (as shown) or for $\mathrm{CF}<22 \mathrm{kHz}$ and $\mathrm{CF} \geq 22 \mathrm{kHz}$ separately (not shown). In each plot, $\mathrm{n}$ indicates the number of multi-unit recordings for the pre-injection time point; all recordings included the pre-injection time point and at least one post-injection time point. Values of $n$ for each of the six time points were: noise-exposed, vehicle injection, $n=46,44$, 46, 30, 38, 28; noise-exposed, AUT00063 injection, $n=49,45,48,42,29,21$; unexposed, vehicle injection, $n=36,36,15,9,9,9$; unexposed, AUT00063 injection, $n=41,41,41,41$, $27,28$.

Figure 6. Evidence for effects of AUT00063 on the magnitude of noise-evoked responses in IC multi-unit recordings. All plots show mean \pm s.e.m. across recordings; colour scheme and conventions as in Figs. 4 and 5. a) Change in mean firing rate evoked by a $200 \mathrm{~ms} 80 \mathrm{~dB}$ SPL broadband noise relative to pre-injection baseline, for multi-unit recordings with CF $<22$ $\mathrm{kHz}$. Noise-evoked firing rates relative to pre-injection baseline were significantly lower following AUT00063 injection than vehicle injection at the $[30,50]$ and $[60,80]$ time points in noise-exposed animals (Wilcoxon rank-sum test). b) Same analysis for multi-unit recordings with $\mathrm{CF} \geq 22 \mathrm{kHz}$, where significant effects of AUT00063 injection were observed at the $(0,20]$ and $[60,80]$ time points in noise-exposed animals. In all plots, $\mathrm{n}$ indicates the number of multi-unit recordings analysed in each condition for the pre-injection time point; all recordings included the pre-injection time point and at least one post-injection time point. For $\mathrm{CF}<22 \mathrm{kHz}(\mathrm{a})$, values of $\mathrm{n}$ for each of the six time points were: noise-exposed, vehicle 
injection, $\mathrm{n}=23,23,23,23,23,8$; noise-exposed, AUT00063 injection, $\mathrm{n}=20,20,20,16$, 16, 16; unexposed, vehicle injection, $\mathrm{n}=12,12,12,12,7,7$; unexposed, AUT00063 injection, $n=21,21,21,21,12,6$. For $C F \geq 22 \mathrm{kHz}(b)$, values of $n$ for each of the six time points were: noise-exposed, vehicle injection, $n=7,7,7,7,7,0$; noise-exposed, AUT00063 injection, $n=11,11,11,4,4$, 4; unexposed, vehicle injection, $n=8,8,8,8,8$, 8; unexposed, AUT00063 injection, $\mathrm{n}=23,23,23,23,17,9$. 


\section{References}

Ahlf S, Tziridis K, Korn S, Strohmeyer I, Schulze H (2012) Predisposition for and prevention of subjective tinnitus development. PLoS One 7:e44519.

Auerbach BD, Rodrigues PV, Salvi RJ (2014) Central gain control in tinnitus and hyperacusis. Front Neurol 5:206.

Brotherton H, Plack CJ, Maslin M, Schaette R, Munro KJ (2015) Pump up the volume: could excessive neural gain explain tinnitus and hyperacusis? Audiol Neurootol 20:273-282.

Brown MR, El-Hassar L, Zhang Y, Alvaro G, Large CH, Kaczmarek LK (2016) Physiological modulators of Kv3.1 channels adjust firing patterns of auditory brain stem neurons. J Neurophysiol 116:106-121.

Brozoski TJ, Bauer CA, Caspary DM (2002) Elevated fusiform cell activity in the dorsal cochlear nucleus of chinchillas with psychophysical evidence of tinnitus. J Neurosci 22:2383-2390.

Chambers AR, Pilati N, Balaram P, Large CH, Kaczmarek LK, Polley DB (2017)

Pharmacological modulation of Kv3.1 mitigates auditory midbrain temporal processing deficits following auditory nerve damage. Sci Rep 7:17496.

Chrostowski M, Yang L, Wilson HR, Bruce IC, Becker S (2011) Can homeostatic plasticity in deafferented primary auditory cortex lead to travelling waves of excitation? J Comput Neurosci 30:279-299.

Coomber B, Berger JI, Kowalkowski VL, Shackleton TM, Palmer AR, Wallace MN (2014). Neural changes accompanying tinnitus following unilateral acoustic trauma in the guinea pig. Eur J Neurosci 40:2427-2441.

Dehmel S, Pradhan S, Koehler S, Bledsoe S, Shore S (2012) Noise overexposure alters long-term somatosensory-auditory processing in the dorsal cochlear nucleus-possible basis for tinnitus-related hyperactivity? J Neurosci 32:1660-1671.

Diehl KH, Hull R, Morton D, Pfister R, Rabemampianina Y, Smith D, Vimal JM, van de Vorstenbosch C; European Federation of Pharmaceutical Industries Association and 
European Centre for the Validation of Alternative Methods (2001) A good practice guide to the administration of substances and removal of blood, including routes and volumes. J Appl Toxicol 2:15-23.

Eggermont JJ, Roberts LE (2015) Tinnitus: animal models and findings in humans. Cell Tissue Res 361:311-336.

Engineer ND, Riley JR, Seale JD, Vrana WA, Shetake JA, Sudanagunta SP, Borland MS, Kilgard MP (2011) Reversing pathological neural activity using targeted plasticity. Nature 470:101-104.

Glait L, Fan W, Stillitano G, Sandridge S, Pilati N, Large C, Alvaro G, Kaltenbach JA. Effects of AUT00063, a Kv3.1 channel modulator, on noise-induced hyperactivity in the dorsal cochlear nucleus. Hear Res 361:36-44.

Finlayson PG, Kaltenbach JA (2009) Alterations in the spontaneous discharge patterns of single units in the dorsal cochlear nucleus following intense sound exposure. Hear Res 256:104-117.

Gan L, Kaczmarek LK (1998) When, where, and how much? Expression of the Kv3.1 potassium channel in high-frequency firing neurons. J Neurobiol. 37:69-79.

Grigg JJ, Brew HM, Tempel BL (2000) Differential expression of voltage-gated potassium channel genes in auditory nuclei of the mouse brainstem. Hear Res 140:77-90.

Hurley LM, Sullivan MR (2012) From behavioral context to receptors: serotonergic modulatory pathways in the IC. Front Neural Circuits 6:58, doi:10.3389/fncir.2012.00058.

Kalappa BI, Brozoski TJ, Turner JG, Caspary DM (2014) Single unit hyperactivity and bursting in the auditory thalamus of awake rats directly correlates with behavioural evidence of tinnitus. J Physiol 592:5065-5078.

Kaltenbach JA, Zacharek MA, Zhang J, Frederick S (2004) Activity in the dorsal cochlear nucleus of hamsters previously tested for tinnitus following intense tone exposure. Neurosci Lett 355:121-125. 
Kaltenbach JA, Godfrey DA, Neumann JB, McCaslin DL, Afman CE, Zhang J (1998) Changes in spontaneous neural activity in the dorsal cochlear nucleus following exposure to intense sound: relation to threshold shift. Hear Res 124:78-84.

Koehler SD, Shore SE (2013) Stimulus timing-dependent plasticity in dorsal cochlear nucleus is altered in tinnitus. J Neurosci 33:19647-19656.

Kujawa SG, Liberman MC (2009) Adding insult to injury: cochlear nerve degeneration after "temporary" noise-induced hearing loss. J Neurosci 29:14077-14085.

Li S, Choi V, Tzounopoulos T (2013) Pathogenic plasticity of Kv7.2/3 channel activity is essential for the induction of tinnitus. Proc Natl Acad Sci USA 110:9980-9985.

Li W, Kaczmarek LK, Perney TM (2001) Localization of two high-threshold potassium channel subunits in the rat central auditory system. J Comp Neurol. 20:196-218.

Leão K.E., Leão R.N., Deardorff A.S., Garrett A., Fyffe R., Walmsley B (2010). Sound stimulation modulates high-threshold $\mathrm{K}$ currents in mouse auditory brainstem neurons. Eur. J. Neurosci. 32:1658-1667.

Lobarinas E, Dalby-Brown W, Stolzberg D, Mirza NR, Allman BL, Salvi R (2011) Effects of the potassium ion channel modulators BMS-204352 Maxipost and its R-enantiomer on salicylate-induced tinnitus in rats. Physiol Behav 104:873-879.

Manzoor N, Licari FG, Klapchar M, Elkin R, Gao Y, Kaltenbach JA (2012) Noise-induced hyperactivity in the inferior colliculus: its relationship with hyperactivity in the dorsal cochlear nucleus. J Neurophysiol 108:976-988.

Manzoor NF, Gao Y, Licari F, Kaltenbach JA (2013) Comparison and contrast of noiseinduced hyperactivity in the dorsal cochlear nucleus and inferior colliculus. Hear Res 295:114-123.

Mulders WH, Robertson D (2009) Hyperactivity in the auditory midbrain after acoustic trauma: dependence on cochlear activity. Neuroscience 164:733-746. 
Mulders WH, Barry KM, Robertson D (2014) Effects of furosemide on cochlear neural activity, central hyperactivity and behavioural tinnitus after cochlear trauma in guinea pig. PLoS One 9:e97948.

Mulders WH, Ding D, Salvi R, Robertson D (2011) Relationship between auditory thresholds, central spontaneous activity, and hair cell loss after acoustic trauma. J Comp Neurol 519:2637-2647.

Norena AJ (2011) An integrative model of tinnitus based on a central gain controlling neural sensitivity. Neurosci Biobehav Rev 35:1089-1109.

Norena AJ (2015) Revisiting the cochlear and central mechanisms of tinnitus and therapeutic approaches. Audiol Neurootol 20 Suppl 1:53-59.

Perney TM, Marshall J, Martin KA, Hockfield S, Kaczmarek LK (1992). Expression of the mRNAs for the Kv3.1 potassium channel gene in the adult and developing rat brain. $\mathrm{J}$ Neurophysiol. 68:756-66.

Pilati N, Large C, Forsythe ID, Hamann M (2012) Acoustic over-exposure triggers burst firing in dorsal cochlear nucleus fusiform cells. Hear Res 283:98-106.

Roberts LE, Eggermont JJ, Caspary DM, Shore SE, Melcher JR, Kaltenbach JA (2010) Ringing ears: the neuroscience of tinnitus. J Neurosci 30:14972-14979.

Robertson D, Bester C, Vogler D, Mulders WH (2013) Spontaneous hyperactivity in the auditory midbrain: relationship to afferent input. Hear Res 295:124-129.

Rudy B, Chow A, Lau D, Amarillo Y, Ozaita A, Saganich M, Moreno H, Nadal MS, Hernandez-Pineda R, Hernandez-Cruz A, Erisir A, Leonard C, Vega-Saenz de Miera E (1999) Contributions of Kv3 channels to neuronal excitability. Ann NY Acad Sci 868:304-343

Schaette R, Kempter R (2006) Development of tinnitus-related neuronal hyperactivity through homeostatic plasticity after hearing loss: a computational model. Eur J Neurosci 23:3124-3138. 
Schaette R, Kempter R (2008) Development of hyperactivity after hearing loss in a computational model of the dorsal cochlear nucleus depends on neuron response type. Hear Res 240:57-72.

Schaette R, McAlpine D (2011) Tinnitus with a normal audiogram: physiological evidence for hidden hearing loss and computational model. J Neurosci 31:13452-13457.

Shore SE, Koehler S, Oldakowski M, Hughes LF, Syed S (2008) Dorsal cochlear nucleus responses to somatosensory stimulation are enhanced after noise-induced hearing loss. Eur J Neurosci 27:155-168

Si-qiong JL and Kaczmarek L (1998) The expression of two splice variants of the Kv3.1 potassium channel gene is regulated by different signaling pathways. J Neurosci 18:2881-90.

Strumbos JG, Polley DB, Kaczmarek LK (2010) Specific and rapid effects of acoustic stimulation on the tonotopic distribution of Kv3.1b potassium channels in the adult rat. Neuroscience 167:567-572.

Vogler DP, Robertson D, Mulders WH (2011) Hyperactivity in the ventral cochlear nucleus after cochlear trauma. J Neurosci 31:6639-6645.

Vogler DP, Robertson D, Mulders WH (2014) Hyperactivity following unilateral hearing loss in characterized cells in the inferior colliculus. Neuroscience 265:28-36.

von Hehn CA, Bhattacharjee A, Kaczmarek LK (2004) Loss of Kv3.1 tonotopicity and alterations in cAMP response element-binding protein signaling in central auditory neurons of hearing impaired mice. J Neurosci 24:1936-1940.

Wu C, Martel DT, Shore SE (2016) Increased synchrony and bursting of dorsal cochlear nucleus fusiform cells correlate with tinnitus. J Neurosci 36:2068-2073. 

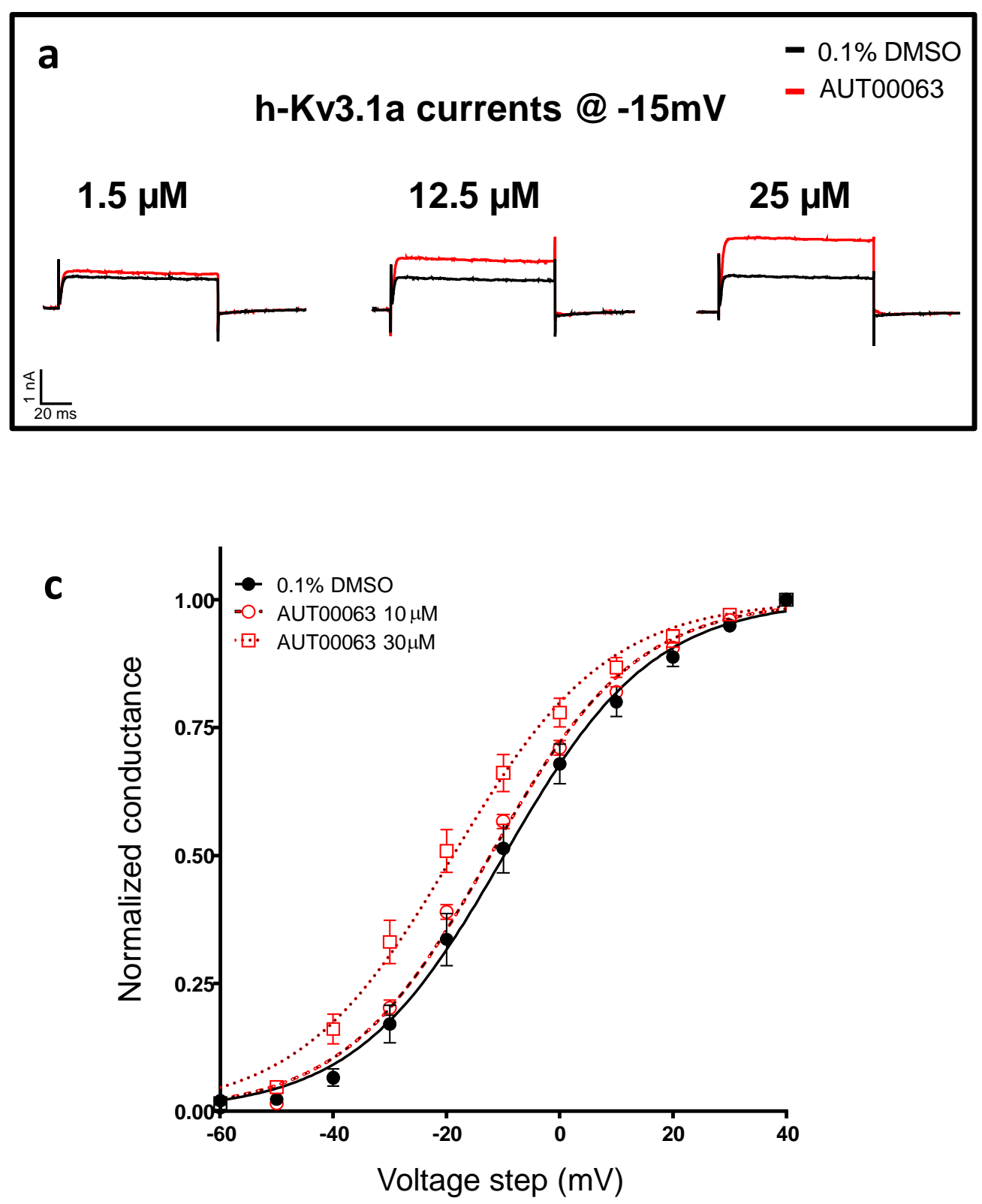

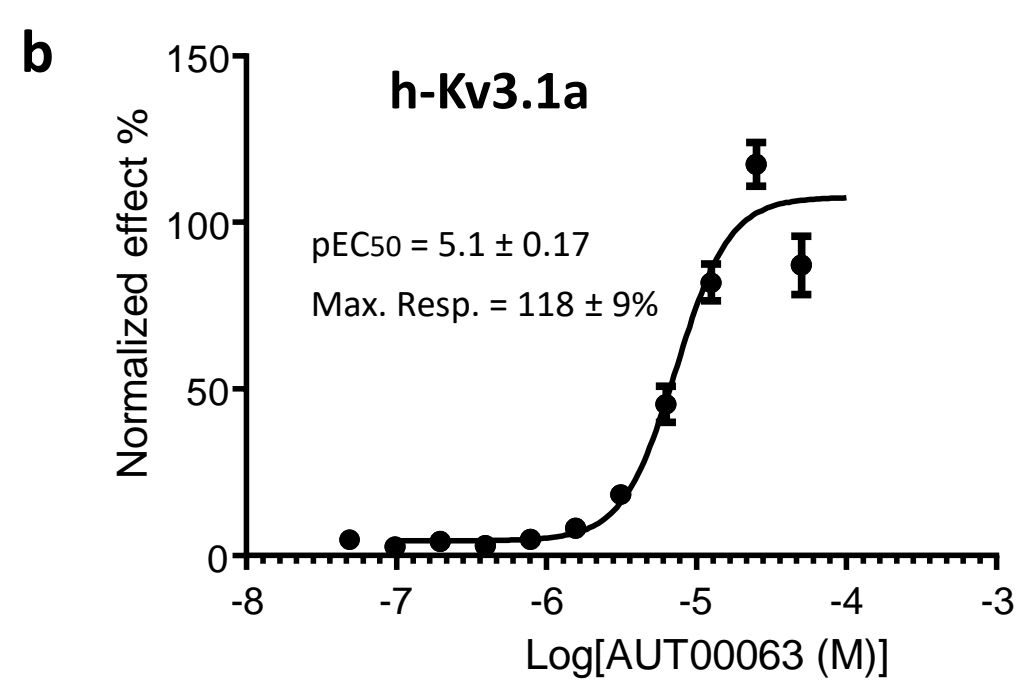

d

$\Delta$ Deactivation $\tau @-40 m V$ (pre-pulse +40mV)

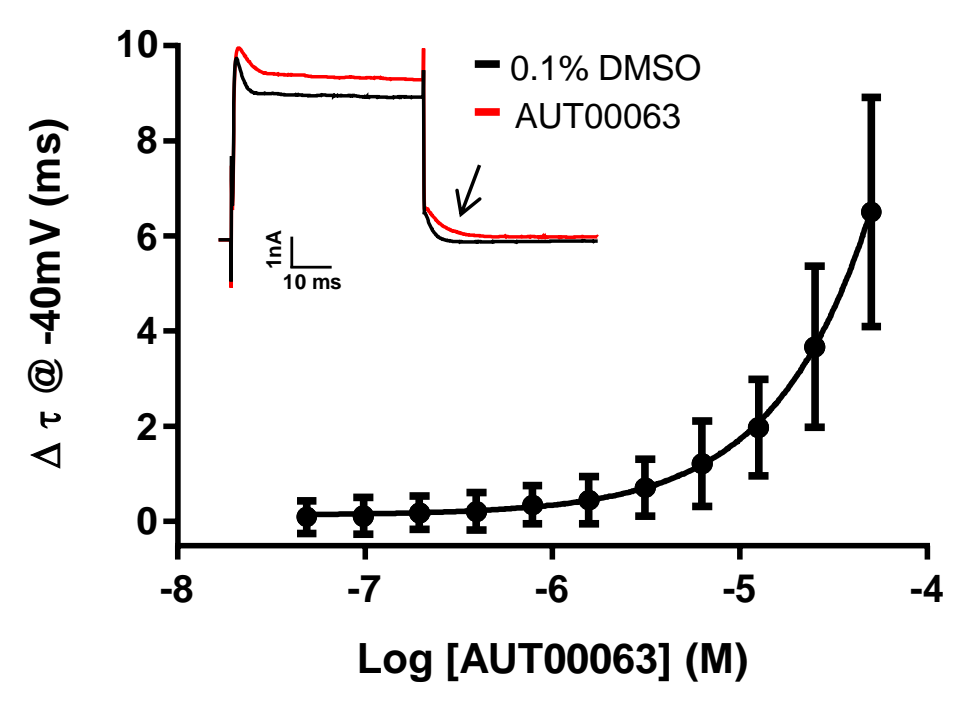




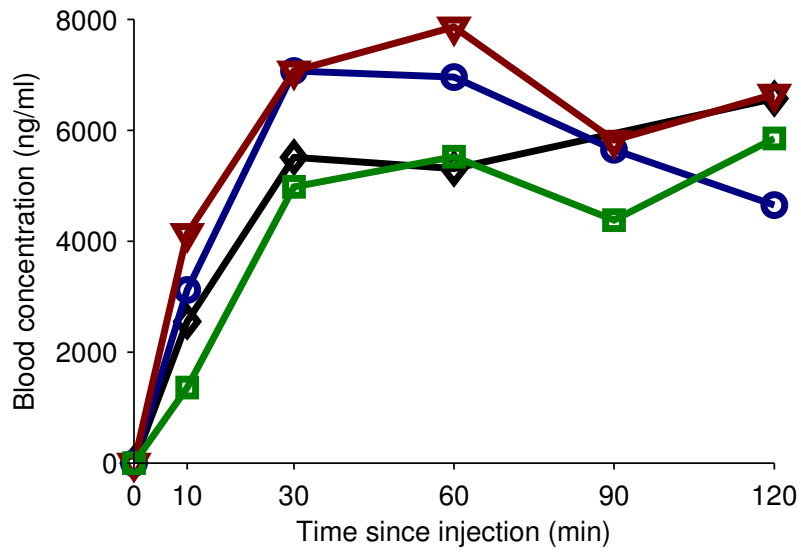




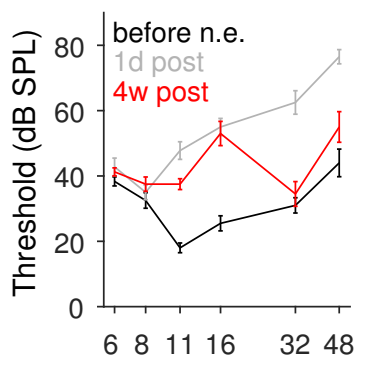

Frequency $(\mathrm{kHz})$$$
\text { . }
$$

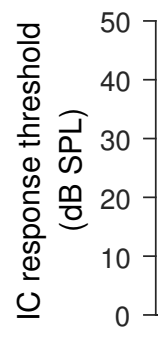

b

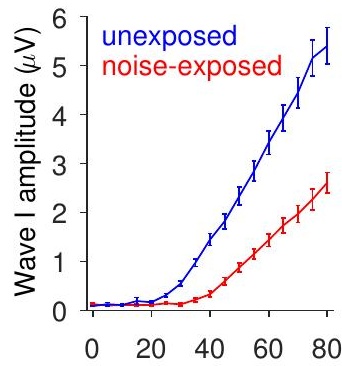

Click level (dB SPL)

unexposed noise-exposed
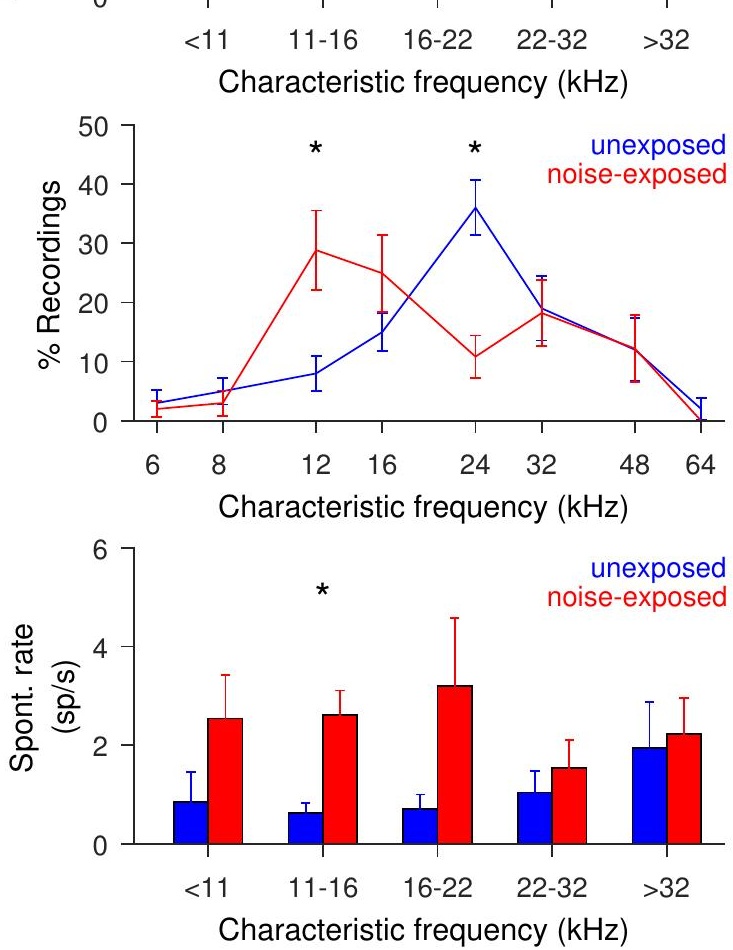
Noise Response Change, CF $<22 \mathrm{kHz}$

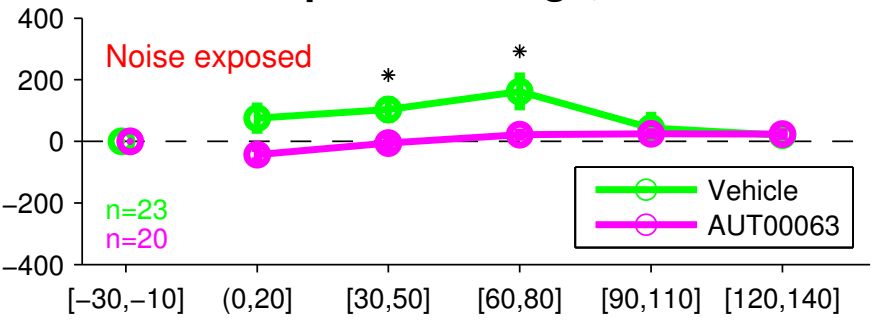

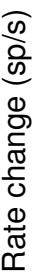

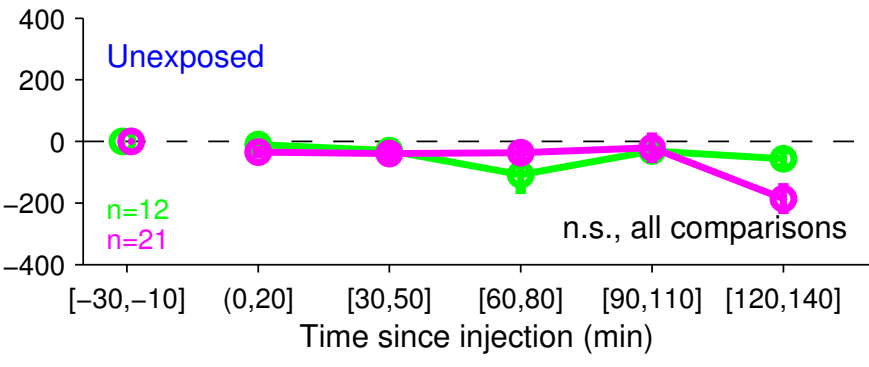

Noise Response Change, CF >= $22 \mathrm{kHz}$

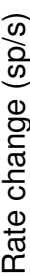

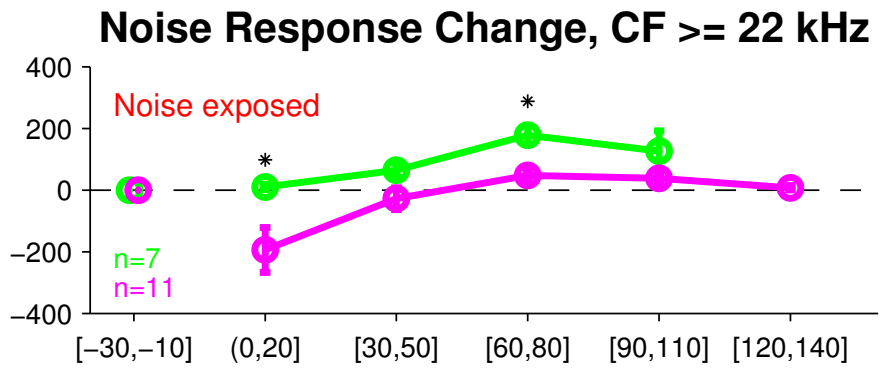

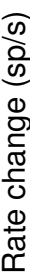

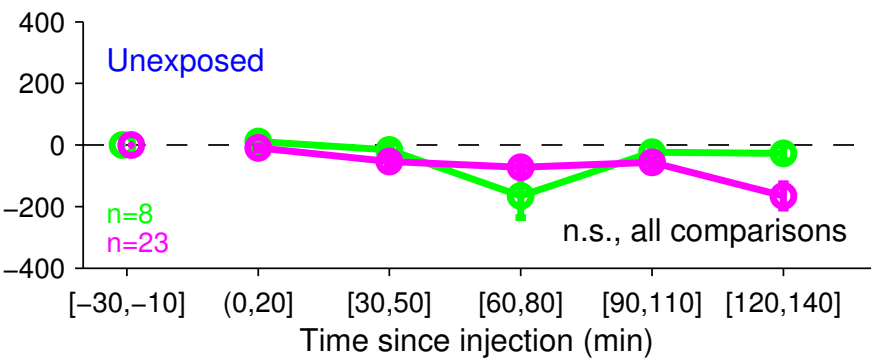

